\title{
Adaptive force transmission in amoeboid cell migration
}

\author{
Jörg Renkawitz ${ }^{1}$, Kathrin Schumann ${ }^{1}$, Michele Weber ${ }^{1}$, Tim Lämmermann ${ }^{1}$, Holger Pflicke ${ }^{1}$, Matthieu Piel ${ }^{2}$, \\ Julien Polleux ${ }^{3,4}$, Joachim P. Spatz ${ }^{4}$ and Michael Sixt ${ }^{1,5}$
}

The leading front of a cell can either protrude as an actinfree membrane bleb that is inflated by actomyosin-driven contractile forces, or as an actin-rich pseudopodium, a site where polymerizing actin filaments push out the membrane ${ }^{1-3}$. Pushing filaments can only cause the membrane to protrude if the expanding actin network experiences a retrograde counterforce, which is usually provided by transmembrane receptors of the integrin family ${ }^{4}$. Here we show that chemotactic dendritic cells mechanically adapt to the adhesive properties of their substrate by switching between integrin-mediated and integrin-independent locomotion. We found that on engaging the integrin-actin clutch, actin polymerization was entirely turned into protrusion, whereas on disengagement actin underwent slippage and retrograde flow. Remarkably, accelerated retrograde flow was balanced by an increased actin polymerization rate; therefore, cell shape and protrusion velocity remained constant on alternating substrates. Due to this adaptive response in polymerization dynamics, tracks of adhesive substrate did not dictate the path of the cells. Instead, directional guidance was exclusively provided by a soluble gradient of chemoattractant, which endowed these 'amoeboid' cells with extraordinary flexibility, enabling them to traverse almost every type of tissue.

Dendritic cells are prototypic examples of rapidly migrating leukocytes that are able to flexibly navigate through three dimensional (3D) environments without tightly adhering to specific substrates ${ }^{5,6}$. To investigate the principles of cytoskeletal force generation and the transduction of these forces onto the environment, we transfected dendritic cells with Lifeact-GFP, which allows visualization of F-actin without interfering with polymerization dynamics ${ }^{7}$. We then used spinning disc confocal and total internal reflection fluorescence (TIRF) microscopy to track cortical actin dynamics. Using photobleaching, colocalization and fluorescence speckle microscopic approaches, we verified that movement patterns of Lifeact faithfully represented F-actin flow (Supplementary Information, Fig. S1 and Movie 1). We immobilized dendritic cells on coverslips coated with poly-L-lysine, and found that a fraction of cells showed centripetal retrograde actin flow. This steady-state treadmilling of non-polarized cells allowed us to use pharmacological intervention to assess the force components driving cortical actin dynamics. When we exposed cells to the G-actin-sequestering drug latrunculin, polymerization at the cell edge was abruptly stopped, but retrograde flow continued (Fig. 1a, b; Supplementary Information, Movie 2) until the entire actin cortex was pulled into the cell centre, where the F-actin signal vanished after 1-2 min. This indicated that the pushing force of polymerization was not the sole force driving retrograde flow and suggested that myosin motors pull F-actin into the cell centre where it depolymerizes. To test this hypothesis, we treated cells with the myosin II inhibitor blebbistatin and observed that retrograde flow continued, albeit with reduced velocity (Fig. 1c, d). Adding latrunculin to cells pre-treated with blebbistatin completely stalled cortical dynamics and also prevented depolymerization (Fig. 1c, d; Supplementary Information, Movie S3). These experiments suggested that, as in aplysia neuronal growth cones and sea urchin coelomocytes ${ }^{8,9}$ but unlike in most other cell types, such as fibroblasts and epithelial cells ${ }^{10}$, myosin II and actin polymerization are the predominant forces driving cortical actin dynamics in dendritic cells. Both forces produce retrograde flow and hence represent the potential intracellular forces that, once polarized and transmitted to a substrate, can power migration.

To gain insight into the actual locomotion process, we mapped actin and myosin II in dendritic cells migrating along gradients of the chemokine CCL19, which guides their in vivo migration from the skin to the draining lymph node ${ }^{11}$. To provide the confined environment these cells need for adhesion-independent movement, we used a setup whereby cells migrated between a layer of agarose and a coverslip ${ }^{12,13}$ (Supplementary Information, Fig. S1). Here, dendritic cells formed a broad leading edge with a diffusely protruding actin cortex and a trailing edge with thicker actin bundles (Fig. 1e; Supplementary Information, Movie 4). Kymograph analysis revealed that at the leading edge of migrating cells, actin remained stationary in relation to the substrate when measured along the central length axis of the cell (Fig. 1f). At the lateral edges of the cell, actin at the front slid inwards, whereas towards the trailing edge it gradually started to glide forwards in relation to the substrate

${ }^{1}$ Max Planck Institute of Biochemistry, Hofschneider Group Leukocyte Migration, 82152 Martinsried, Germany. ${ }^{2}$ CNRS UMR144, Institut Curie, Paris, France. ${ }^{3}$ Max Planck Institute of Biochemistry, Department of Molecular Medicine, 82152 Martinsried, Germany. ${ }^{4}$ Max Planck Institute of Metal Research, Department of New Materials and Biosystems, 70569 Stuttgart, Germany.

${ }^{5}$ Correspondence should be addressed to M.S. (e-mail: sixt@biochem.mpg.de).

Received 27 August 2009; accepted 21 October 2009; published online 15 November 2009; DOI: 10.1038/ncb1992 
a
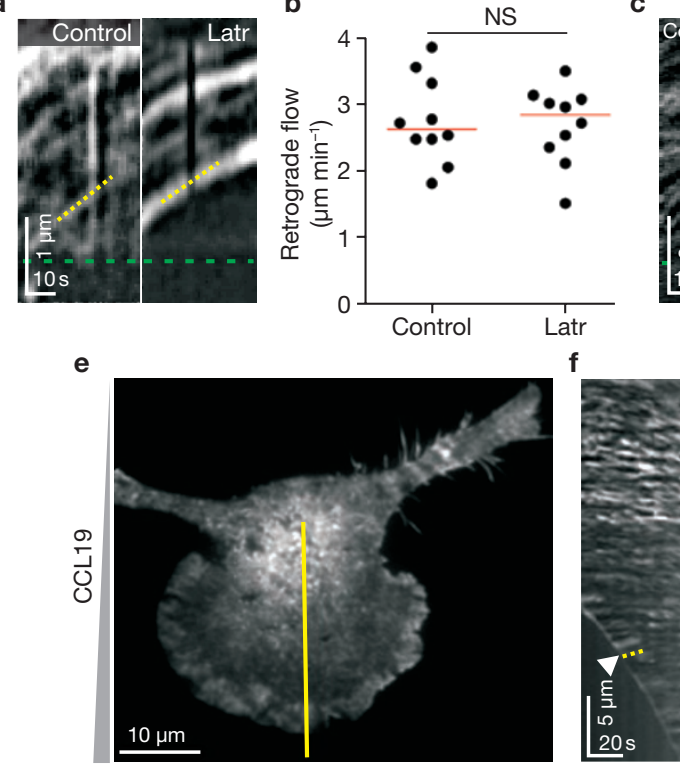

Figure 1 Spatial and temporal dynamics of actin and myosin II in migrating dendritic cells. (a) Lifeact-GFP-transfected dendritic cells adhere to poly-L-lysine coated surfaces. Kymographic analysis of retrograde actin flow (dashed yellow lines) before (Control) and after addition of latrunculin B (Latr; 500 nM). (b) Quantification of retrograde flow velocities from ten different regions in one representative cell out of three. Means are depicted as red lines. (c) Kymographic analysis was performed as in a before and after addition of blebbistatin ( $\mathrm{Bl} ; 50 \mu \mathrm{M}$, ) and then latrunculin (500 nM). Dashed green lines in $\mathbf{a}$ and $\mathbf{c}$ represent the leading edge. (d) Quantitative analysis of $\mathbf{c}$ (from 10 different regions in one representative cell out of three). Means are depicted as red lines, ${ }^{* * *} P<0.001$. To exclude possible

(Supplementary Information, Movie 4). To functionally localize the site of actin polymerization, we performed photobleaching experiments with actin-GFP-transfected cells. These revealed that actin recovered from the cell front (Supplementary Information, Figs S2-4 and Movie 5), indicating that nucleation of barbed ends is largely restricted to the leading edge. To assess whether the plasma membrane shows the same flow pattern, we used the fluorescent lipid dye DiO to label membrane lipids ${ }^{14}$. After bleaching the leading edge, we could not find any indication of preferential recovery from the cell front (Supplementary Information, Fig. S5 and Movie 6). These experiments argued against bulk membrane flow and were therefore consistent with previous studies suggesting that rather than being extensively remodelled and continuously transported to the cell front, the membrane behaves like a sliding bag that is pushed forward by the underlying cytoskeleton ${ }^{14-16}$. To identify the sites of potential actomyosin contraction, we tracked the localization of myosin light chain (MLC)-GFP, which tags myosin II. MLC localized in patches with a density gradient increasing from the cell front to the rear (Fig. 1g; Supplementary Information, Movie 7). The leading edge was completely devoid of signal and patches started to materialize $5-10 \mu \mathrm{m}$ behind the leading front (Fig. 1g, h). The movement of MLC-GFP followed the same principal trajectories as F-actin, and patches gradually converged at the trailing edge, reflecting their contractile activity (Supplementary Information, Fig. S6). Together, these data suggest that force generated by the actin network that grows below the leading plasma membrane simultaneously causes the membrane to protrude and pushes the actin cortex backwards. Myosin II contracts the network in the posterior regions and thereby imposes a pulling force on the anterior network. d
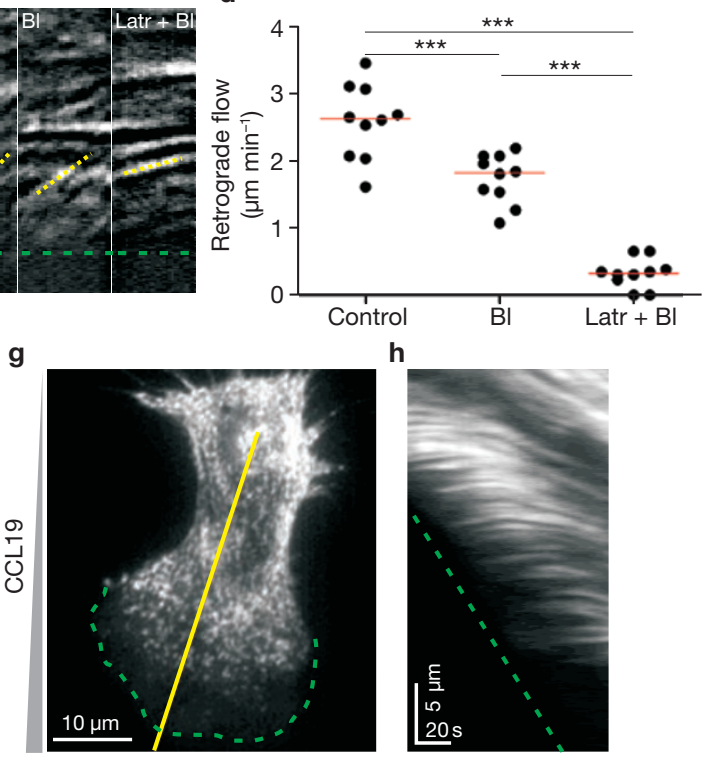

phototoxic effects when using blebbistatin, the same experiments were performed using endpoint analysis, and these showed comparable results (data not shown). (e) Lifeact-GFP-transfected dendritic cells migrating under agarose in a CCL19 gradient. (f) Kymographic analysis of yellow line in e. The short zone of retrograde actin flow at the leading edge is highlighted by a dashed yellow line and an arrowhead. (g) MLC-GFPtransfected dendritic cells migrating under agarose in a CCL19 gradient. (h) Kymographic analysis of yellow line in $\mathrm{g}$. The leading edges of the cells are highlighted with a dashed green line in $\mathbf{g}$ and $\mathbf{h}$. All cells were imaged using TIRF microscopy. For statistical analysis, see Supplementary Information, Table S1. NS, not significant.

The fact that we observed almost no retrograde flow in migrating dendritic cells pointed to force coupling at the leading edge, preventing retrograde flow and converting polymerization entirely into protrusion. When plated on 2D surfaces, mature dendritic cells do not attach to substrates, meaning that they move without or with very low adhesive forces ${ }^{17}$. We performed interference reflection microscopy to visualize membranesubstrate proximity (an indicator of adhesive interaction) under agarose and observed a band of destructive interference at the cell front, suggesting tight apposition between membrane and substrate (Fig. 2a, b). To test whether integrins or alternative receptors constitute the putative clutch, we used dendritic cells that lacked all integrin heterodimers $\left(\mathrm{Itg}^{-1-}\right.$ dendritic cells). Notably, these cells showed constructive interference at the cell front, indicating that they lacked an adhesive zone (Fig. 2c, d). Despite this release of substrate contact, $\mathrm{Itg}^{-1-}$ dendritic cells reached normal migration velocities in CCL19 gradients (Fig. 2k; Supplementary Information, Fig. S1), which is consistent with our previous findings showing that within confined environments integrin-mediated force coupling is dispensable for locomotion ${ }^{5}$. The seemingly contradictive results of adhesion loss and unimpaired protrusion rates raised the question of whether the potentially disengaged clutch and the resulting loss of retrograde resistance is compensated for by changes in leading-edge actin dynamics. Indeed, when Lifeact-GFP-transfected Itg $^{-1-}$ cells were imaged with TIRF microscopy, a substantial retrograde flow of F-actin in the axis of migration was observed (Fig. 2e, f; Supplementary Information, Movie 8). Kymographic quantification revealed that out of $29 \mathrm{Itg}^{-1-}$ dendritic cells analysed all showed retrograde flow with velocities of up to $20 \mu \mathrm{m} \mathrm{min}^{-1}$, whereas only $8 \%$ of wild-type cells had maximum flow rates of $5 \mu \mathrm{m} \mathrm{min}^{-1}$ (Fig. $2 \mathrm{~g}$; for 

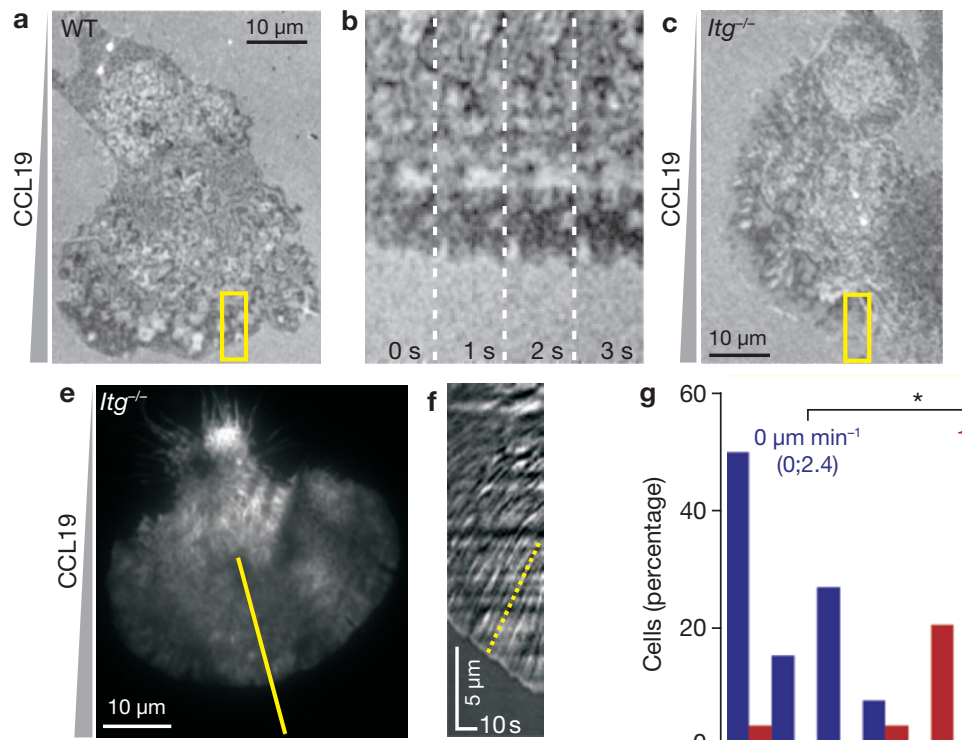
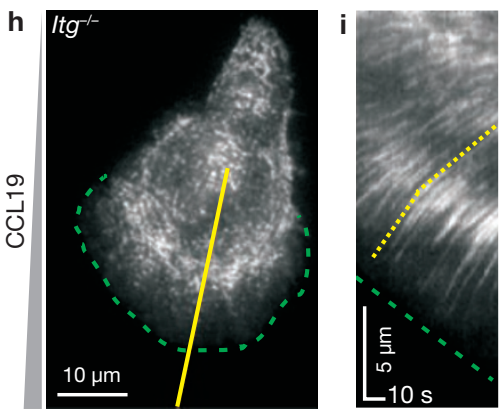

Figure 2 Dendritic cells can use integrins for transmembrane force coupling, and uncoupling enhances retrograde actin flow without affecting migration. (a) Interference reflection microscopy (IRM) image of a migrating wild-type dendritic cell. (b) Image sequence of enlarged, boxed region in a. (c) IRM image of a migrating integrin-deficient ( $\left./ \mathrm{tg}^{-/}\right)$dendritic cell. (d) Image sequence of enlarged boxed region in c. (e) TIRF image of a Lifeact-GFP-transfected Itg $^{-1}$ dendritic cell. (f) Kymographic analysis of yellow line in e. The retrograde actin flow is highlighted by a dashed yellow line. (g) Quantitative analysis of retrograde flow velocities; $n(\mathrm{WT})=33$, $n\left(/ \mathrm{tg}^{-1}\right)=29$. (h) TIRF image of a MLC-GFP-transfected $/ \mathrm{tg}^{-/}$dendritic cell. (i) Kymographic analysis of yellow line in $\mathbf{h}$. The retrograde actin flow

statistics see Supplementary Information, Tables S1, S2). To exclude the possibility that increased retrograde flow in uncoupled cells was caused by enhanced contractility, we measured actin flow in $\mathrm{Itg}^{-1-}$ cells treated with blebbistatin and found velocities comparable to untreated $\mathrm{Itg}^{-1-}$ cells (Supplementary Information, Fig. S6). Also, MLC patches, which did not show any retrograde movement in wild-type cells, slid backwards in Itg-1dendritic cells (Fig. 2h-j; Supplementary Information, Movie 9). Normal cell-shape, continuous actin cortex protruding with unaltered velocity (Fig. $2 \mathrm{k}$ ) and increased retrograde actin flow implied that polymerization was accelerated in the absence of integrins. Accordingly, the polymerization rate, measured as the sum of protrusion and retrograde flow, increased from a mean of $9.1 \mu \mathrm{m} \mathrm{min}{ }^{-1}$ ( $\pm 0.7 \mathrm{~s} . \mathrm{d}$.) in wild-type cells to $17.8 \mu \mathrm{m} \mathrm{min}^{-1}$ ( \pm 0.7 s.d.) in Itg $^{-/-}$dendritic cells (Fig. 2l).

Retrograde actin flow $\left(\mu \mathrm{m} \mathrm{min}^{-1}\right)$
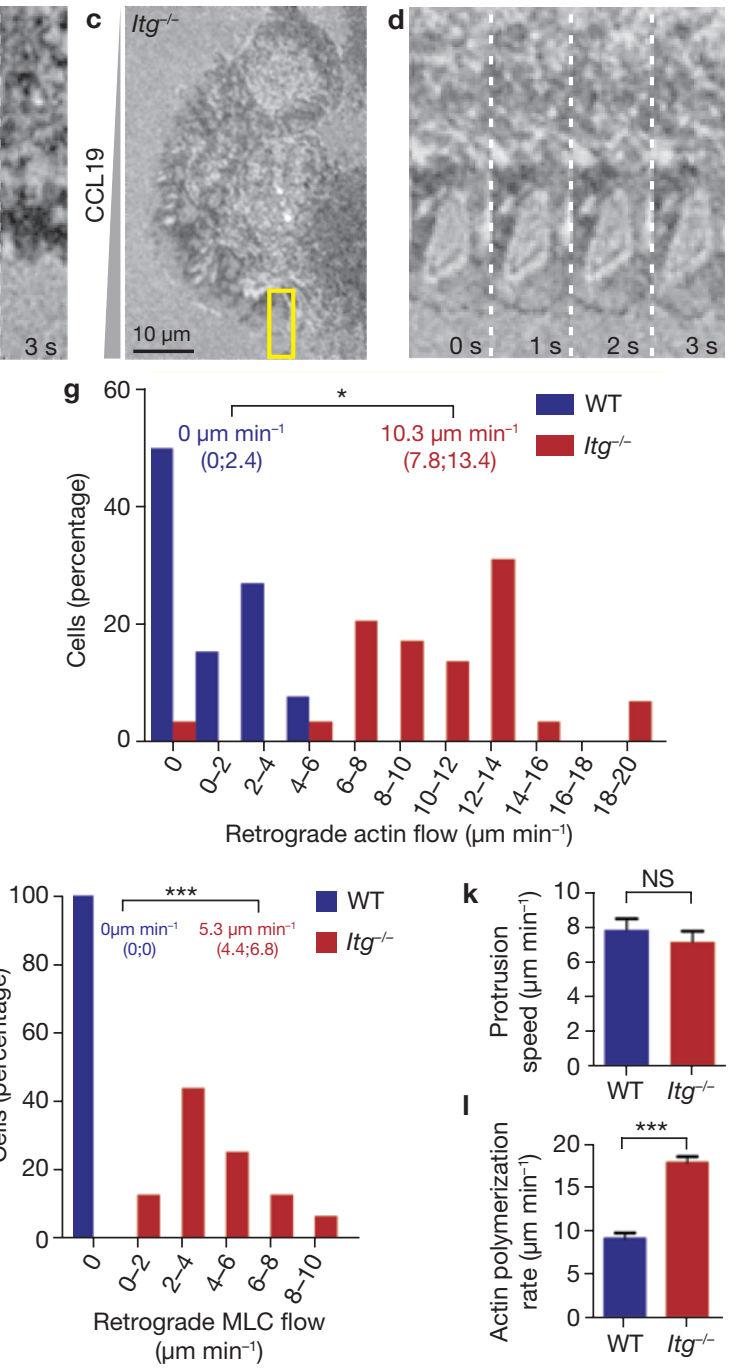

is highlighted by a dashed yellow line. The leading edges of the cells are highlighted with a dashed green line in $\mathbf{h}$ and $\mathbf{i}$. (j) Quantitative analysis of retrograde MLC flow; $n(\mathrm{WT})=6, n\left(\mathrm{Itg}^{-1}\right)=16$. (k) Protrusion velocity in wild-type and $\mathrm{Itg}^{-1-}$ dendritic cells. Error bars indicate mean \pm s.e.m., $n(\mathrm{WT})=33, n\left(\mathrm{Itg}^{-1}\right)=29$. (I) Actin polymerization rate in wild-type and $\mathrm{Itg}^{-1-}$ dendritic cells. Error bars indicate mean \pm s.e.m., $n(\mathrm{WT})=33, n$ $\left(/\right.$ tg $\left.^{-1}\right)=29$. All cells migrate under agarose in CCL19 gradients. ${ }^{*} P<$ $0.05, * * * P<0.001$. Numbers in $\mathbf{g}$ and $\mathbf{j}$ represent mean values with 25 th and 75 th percentiles in brackets. $n$ values represent number of cells. For statistical analysis, see Supplementary Information, Tables S1 and S2. MLC, myosin-light chain; NS, not significant. WT, wild-type.

To corroborate this finding, we interfered with the molecular clutch, intracellularly at the interface between the actin cortex and integrin and extracellularly at the contact area between integrin and the substrate. For intracellular uncoupling, we used talin $1^{-/-}\left(\mathrm{Tln}^{-/-}\right)$dendritic cells, in which integrins are present on the cell surface, but both actin and substrate linkage is eliminated ${ }^{5}$. Confirming the $\mathrm{Itg}^{-1-}$ results, $\mathrm{Tln}^{-1-}$ dendritic cells showed the same degree of retrograde flow as $\mathrm{Itg}^{-1-}$ dendritic cells, and therefore had increased polymerization rates compared with wild-type cells (Fig. 3a, b and data not shown). We next attempted to abrogate integrin-ligand interactions. The force coupling we observed was exclusively mediated by members of the $\beta 2$ integrin family (Supplementary Information, Fig. S7), and two of these ( $\alpha \mathrm{M} \beta 2$ and $\alpha x \beta 2$ ) bind to charged residues on glass, plastic and proteins ${ }^{18}$. As 

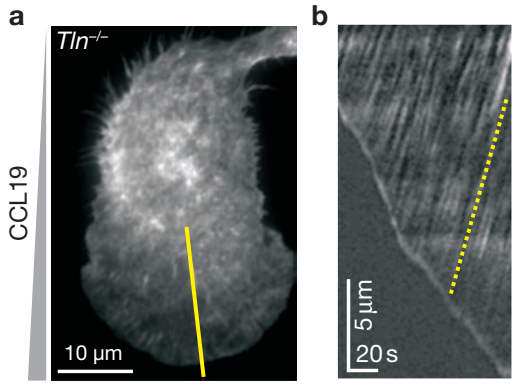

f

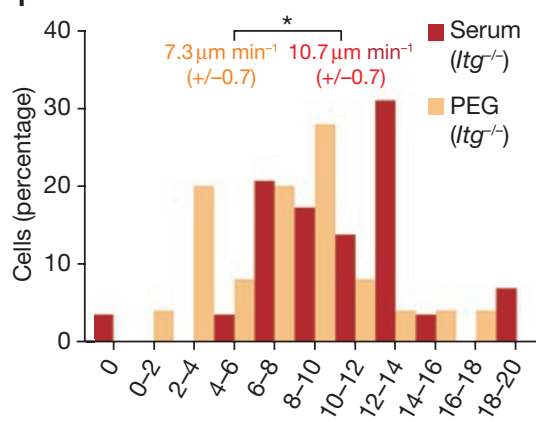

Retrograde actin flow $\left(\mu \mathrm{m} \mathrm{min} \mathrm{m}^{-1}\right)$
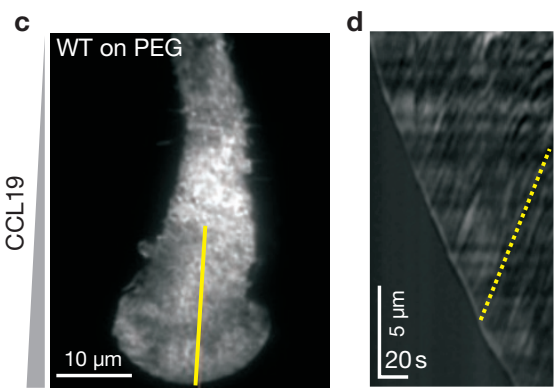

g

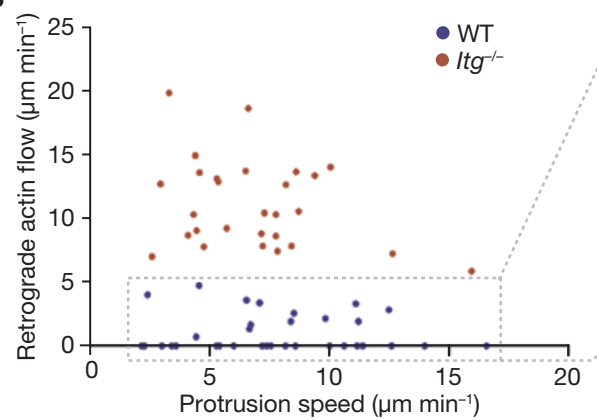

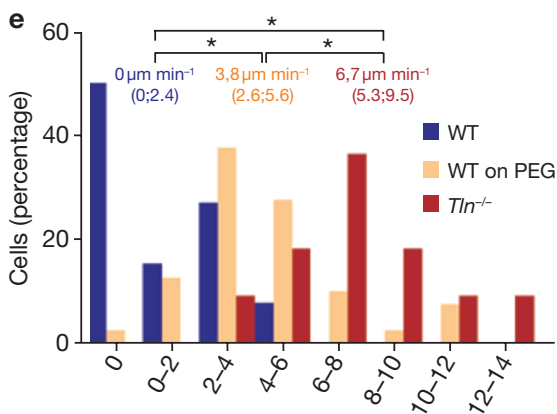

Retrograde actin flow $\left(\mu \mathrm{m} \mathrm{min}^{-1}\right)$

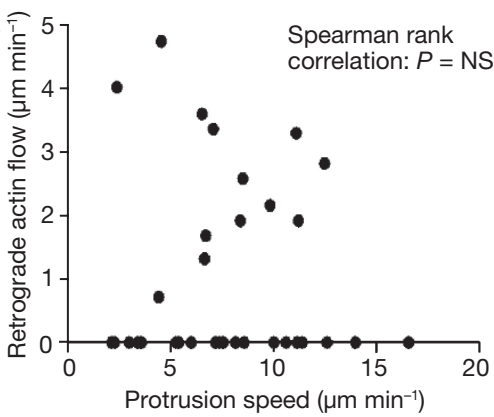

Figure 3 Actin polymerization rate is variable in the molecular clutch system. (a) Lifeact-GFP-transfected talin1-deficient ( $\mathrm{TI}^{-\mathrm{I}^{-}}$) dendritic cell. (b) Kymographic analysis of yellow line in a. The retrograde actin flow is highlighted by a dashed yellow line. (c) Lifeact-GFP-transfected dendritic cells migrating on polyethylene glycol (PEG). (d) Kymographic analysis of yellow line in $\mathbf{d}$. The retrograde actin flow is highlighted by a dashed yellow line. (e, f) Quantitative analysis of retrograde actin flow velocities. Numbers represent mean values with 25 th and 75 th percentiles

this promiscuous binding did not allow us to use specific substrates, we ensured the absence of any immobilized ligands that may have been absorbed from the serum fraction. To this end, we passivated the substrate with polyethylene glycol (PEG), which is inert. As with genetic uncoupling, the migration velocity of wild-type dendritic cells on PEG was unchanged compared with serum-coated surfaces, and retrograde flow was substantially increased (Fig. 3c, d; Supplementary Information, Movie 10). However, flow velocities did not reach that of $\mathrm{Itg}^{-1-}$ and $\mathrm{Tln}^{-1-}$ dendritic cells (Fig. 3e), which is probably because even in the absence of ligand binding, integrins experience friction when pulled through the viscous plasma membrane and thereby represent a retrograde resistance for the actin flow. When $\mathrm{Itg}^{-1-}$ cells were placed on PEG, retrograde flow velocities were comparable to those of $\mathrm{Itg}^{-1-}$ cells on serum-coated surfaces, indicating that no other forcecoupling receptors had an effect, in the setup used (Fig. 3f). To further probe the frictional interface between the actin cortex, membrane and substrate, we coated the surfaces with concanavalinA which binds membrane carbohydrates ${ }^{19}$. As expected, dendritic cells were unable to migrate in this setting as the membrane was 'glued' to the surface (data not shown). Interestingly, It $^{-1-}$, but not wild-type dendritic cells could still polarize and the actin cortex continued to 'run on the spot'. Polymerization rates were similar to those of $\mathrm{Itg}^{-/-}$dendritic cells on serum-coated surfaces (Supplementary Information, Fig. S8), indicating that the friction between the actin cortex and membrane is mainly due to force coupling through integrins. in brackets. $n(\mathrm{WT})=33 ; n(\mathrm{PEG})=40 ; n\left(T / n^{-/-}\right)=29$ (e) and mean values \pm s.e.m., $n($ Serum $)=29 ; n(P E G)=27$ (f). (g) Left, retrograde actin flow in wild-type and integrin-deficient $\left(/ \mathrm{tg}^{-1}\right)$ dendritic cells plotted against the protrusion speed. Right, close-up view of wild-type dendritic cells from boxed region in $\mathbf{g}$. All cells migrated under agarose in CCL19 gradients and were imaged using TIRF microscopy. ${ }^{*} P<0.05$. For statistical analysis, see Supplementary Information, Tables S1 and S2. $n$ values represent number of cells. WT, wild-type. NS, not significant.

In all cases of uncoupling, the retrograde flow penetrated deep into the cell centre where it gradually slowed, eventually stopping in the neck region between the leading and trailing edge (Supplementary Information, Fig. S8). Here, within a narrow area, the flow direction reversed from retrograde at the front to anterograde at the back, indicating that in this convergence zone the actin cortex experienced a counterforce, which is probably the actin cortex itself as it is more extensively crosslinked in posterior regions. We concluded that, once the molecular clutch at the leading edge is disengaged, the combined force of actin polymerization and contraction lose their retrograde counter-force and cause cortical slippage.

Unexpectedly, the increased retrograde flow neither caused obvious shape changes of the leading edge nor decreased protrusion velocities, as it was accompanied by an increased actin polymerization rate. One possible explanation for this feedback between force coupling and polymerization rate is entirely mechanistic: given that membrane tension stays constant ${ }^{15}$, the growth of a backward slipping actin cortex is subjected to less counter-force at the membrane. According to the well-established force-velocity relationship of polymerizing networks ${ }^{3,20}$ and the fact that G-actin is available in excess ${ }^{21}$, the G-actin incorporation rate increases until a new equilibrium is reached under which membrane tension and hence protrusion speed are maintained. To test whether this is a dendritic cell-specific phenomenon, we measured polymerization rates in macrophages placed on PEG versus serum-coated surfaces and found significantly increased rates on 

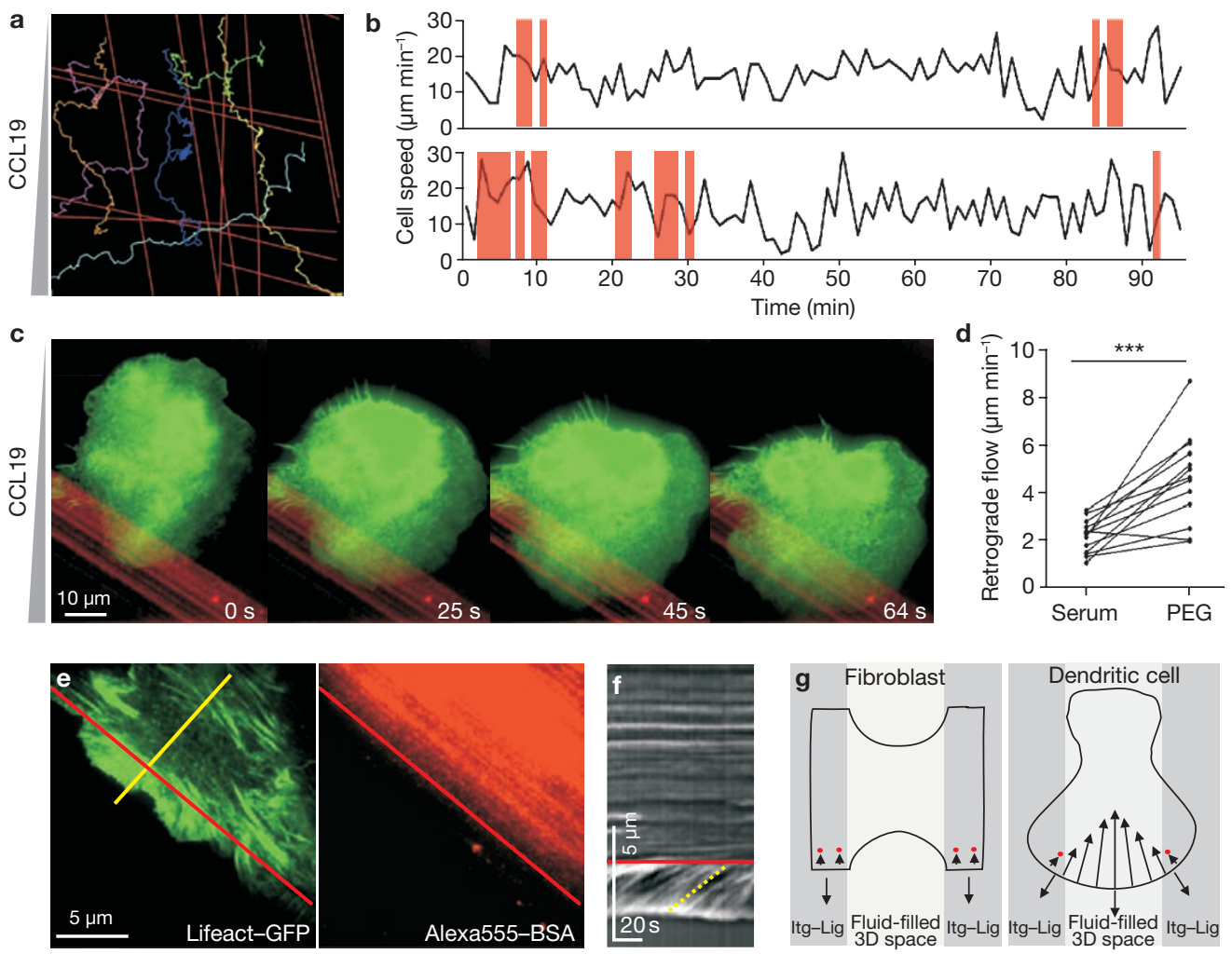

Figure 4 Leading edge protrusion and chemotactic movement is substrateindependent. (a) Migratory tracks (coloured, jagged lines) of dendritic cells migrating under agarose in a CCL19 gradient on a substrate containing adhesive tracks (red lines; visualized by red fluorescent bovine serum albumin) surrounded by an inert polyethylene glycol (PEG) surface (black areas).

(b) Velocity of the cells corresponding to the orange (upper panel) and yellow (lower panel) lines in a, either on an adhesive substrate (red background) or on an inert PEG surface (white background). (c) Close-up view of a Lifeact-GFPtransfected cell from the assay described in a. (d) Comparative analysis of the retrograde actin flow within cells ( $n=12$ cells) in simultaneous contact with serum- and PEG-coated areas ( $\left.{ }^{* * *} P<0.001\right)$. (e) Lifeact-GFP-transfected

PEG, suggesting that such a mechanical response is of broad relevance (Supplementary Information, Fig. S9). This adaptation introduces the actin polymerization rate as a third variable in the molecular clutch concept and predicts that, in dendritic cells, velocities of retrograde flow and protrusion are not necessarily correlated (Supplementary Information, Fig. S9). Indeed, plotting protrusion against retrograde flow velocities did not reveal the inverse relationship that has been observed in neuronal growth-cones and keratocytes ${ }^{9,22-24}$, where cells slow down on disengagement of the clutch (Fig. 3g).

An inherent mechanical adaptation of the actin cortex to the strength of force coupling would imply that dendritic cells can rapidly switch between substrates without requiring the adjustment of global biochemical parameters. To test this prediction, we prepared patterned surfaces containing serum-coated tracks alternating with PEG-coated areas. Tracking of dendritic cells migrating towards CCL19 revealed that the cells migrated equally well, and without preference, on both surfaces (Fig. 4a). Migration kinetics were unaffected when cells crossed borders (Fig. 4b), suggesting either a rapid temporal switch or spatial adaptation of the actin cortex at substrate interfaces. To test whether dendritic cells can locally adapt their actin flow, we followed migration on patterns at high magnification. This revealed that cells crossed borders without

fibroblast from the assay described in a. The red lines show the border of the substrate containing adhesive tracks. (f) Kymographic analysis of the yellow line in e. The retrograde actin flow is highlighted by a dashed yellow line and the border of the substrate containing adhesive tracks is highlighted by a red line. (g) Scheme illustrating the difference between surface-anchoring cells (for example, fibroblasts), which protrude exclusively along adhesive substrates, and dendritic cells, which are able to protrude into open space. Red dots represent adhesion sites, downward arrows represent protrusion direction and upward arrows represent retrograde actin flow. Itg-Lig, integrin-ligand. $n$ values represent number of cells. For statistical analysis, see Supplementary Information, Table S1.

obvious morphological change, except that occasional and transient retraction fibres formed at the trailing edge when leaving the adhesive area (data not shown). TIRF imaging of actin flow revealed that within the same cell, retrograde flow was increased on PEG compared with serum-coated areas (Fig. 4c, d), indicating the capability of dendritic cells to locally adjust their actin flow.

We demonstrated that local adaptation of the actin polymerization rate steadily causes the leading membrane of dendritic cells to protrude, irrespective of whether retrograde flow is counteracted by a transmembrane clutch or is sliding into an internal resistance. As such mechanical adaptation should be a universal feature of the cytoskeleton, we placed fibroblasts onto patterned substrates under agarose. As shown previously ${ }^{25}$, and in contrast to leukocytes, these cells could not maintain a polarized shape on PEG, and they adapted their shape strictly to the pattern of the adhesive surface. However, we noticed that most fibroblasts protruded $2-3 \mu \mathrm{m}$ beyond the pattern edge. Notably, these protrusions showed a massive retrograde actin flow that stopped abruptly at the substrate border (Fig. 4e, f; Supplementary Information, Movie 11). This indicates that increased polymerization is a universal response to decreased force coupling and suggests that counter-forces at the leading membrane usually limit polymerization rates. However, 
it is only in amoeboid cells that increased polymerization can efficiently 'compensate' for slippage caused by inefficient force coupling and thereby enable the cell to send pseudopodia into a fluid-filled interstitial space or beyond slippery substrates (both represented by PEG-coated areas in our assays). What determines such autonomy rather than substrate dependency remains to be determined. One plausible factor might be the differential rigidity of the actin cortex, which allows it to brace against itself in amoeboid cells, while in adherent cells, adhesion sites are required to maintain stability. In conclusion, we show that amoeboid protrusions unify two seemingly mutually exclusive migration models: similarly to membrane blebs they can autonomously extend into open space without requiring mechanical anchorage (Fig. 4g), but unlike blebs, and similar to adherent lamellipodia, they can generate traction by transmitting the retrograde force of actin polymerization to a substrate.

\section{METHODS}

Methods and any associated references are available in the online version of the paper at http://www.nature.com/naturecellbiology/.

Note: Supplementary Information is available on the Nature Cell Biology website.

\section{ACKNOWLEDGEMENTS}

We thank S. Cremer for statistical analysis, K. Hirsch for technical assistance, D. Critchley for talin1-deficient mice and R. Fässler for integrin-deficient mice, discussions and critical reading of the manuscript. This work was supported by the German Research Foundation, the Peter Hans Hofschneider Foundation for Experimental Biomedicine, the Max Planck Society, the Alexander von Humboldt Foundation and the allergology programme of the Landesstiftung Baden-Württemberg.

\section{AUTHOR CONTRIBUTIONS}

J.R. and M.S. designed, performed and analysed experiments and wrote the manuscript. K.S., M.W., T.L. and H.P. performed experiments, M.P. contributed ideas and J.P. and J.P.S. provided passivated surfaces.

\section{COMPETING FINANCIAL INTERESTS}

The authors declare no competing financial interests.

Published online at http://www.nature.com/naturecellbiology/ Reprints and permissions information is available online at http://npg.nature.com/ reprintsandpermissions/

1. Charras, G. \& Paluch, E. Blebs lead the way: how to migrate without lamellipodia Nature Rev. Mol. Cell Biol. 9, 730-736 (2008).
2. Lammermann, T. \& Sixt, M. Mechanical modes of 'amoeboid' cell migration. Curr. Opin. Cell Biol. 21, 636-644 (2009).

3. Mogilner, A. On the edge: modeling protrusion. Curr. Opin. Cell Biol. 18, 32-39 (2006).

4. Mitchison, T. J. \& Cramer, L. P. Actin-based cell motility and cell locomotion. Cel/ 84, 371-379 (1996)

5. Lammermann, T. et al. Rapid leukocyte migration by integrin-independent flowing and squeezing. Nature 453, 51-55 (2008).

6. Lammermann, T. et al. Cdc42-dependent leading edge coordination is essential for interstitial dendritic cell migration. Blood 113, 5703-5710 (2009).

7. Riedl, J. et al. Lifeact: a versatile marker to visualize F-actin. Nature Methods $\mathbf{5}$, 605-607 (2008).

8. Henson, J. H. et al. Two components of actin-based retrograde flow in sea urchin coelomocytes. Mol. Biol. Cell 10, 4075-4090 (1999).

9. Medeiros, N. A., Burnette, D. T. \& Forscher, P. Myosin II functions in actin-bundle turnover in neuronal growth cones. Nature Cell Biol. 8, 215-226 (2006).

10. Cramer, L. P. Molecular mechanism of actin-dependent retrograde flow in lamellipodia of motile cells. Front. Biosci. 2, d260-270 (1997).

11. Alvarez, D., Vollmann, E. H. \& von Andrian, U. H. Mechanisms and consequences of dendritic cell migration. Immunity 29, 325-342 (2008).

12. Heit, B. \& Kubes, P. Measuring chemotaxis and chemokinesis: the under-agarose cell migration assay. Sci. STKE 2003, PL5 (2003).

13. Malawista, S. E. \& de Boisfleury Chevance, A. Random locomotion and chemotaxis of human blood polymorphonuclear leukocytes (PMN) in the presence of EDTA: PMN in close quarters require neither leukocyte integrins nor external divalent cations. Proc. Natl Acad. Sci. USA 94, 11577-11582 (1997).

14. Lee, J., Gustafsson, M., Magnusson, K. E. \& Jacobson, K. The direction of membrane lipid flow in locomoting polymorphonuclear leukocytes. Science $\mathbf{2 4 7}, \mathbf{1 2 2 9 - 1 2 3 3}$ (1990).

15. Keren, K. et al. Mechanism of shape determination in motile cells. Nature 453, 475480 (2008)

16. Kucik, D. F., Elson, E. L. \& Sheetz, M. P. Cell migration does not produce membrane flow. J. Cell Biol. 111, 1617-1622 (1990).

17. Sixt, M. et al. The conduit system transports soluble antigens from the afferent lymph to resident dendritic cells in the T cell area of the lymph node. Immunity 22, 19-29 (2005).

18. Yakubenko, V. P., Lishko, V. K., Lam, S. C. \& Ugarova, T. P. A molecular basis for integrin aMbeta 2 ligand binding promiscuity. J. Biol. Chem. 277, 48635-48642 (2002).

19. Kucik, D. F., Kuo, S. C., Elson, E. L. \& Sheetz, M. P. Preferential attachment of membrane glycoproteins to the cytoskeleton at the leading edge of lamella. J. Cell Biol. 114, 1029-1036 (1991).

20. McGrath, J. L. et al. The force-velocity relationship for the actin-based motility of Listeria monocytogenes. Curr. Biol. 13, 329-332 (2003).

21. Koestler, S. A. et al. F- and G-actin concentrations in lamellipodia of moving cells PLoS One 4, e4810 (2009).

22. Jurado, C., Haserick, J. R. \& Lee, J. Slipping or gripping? Fluorescent speckle microscopy in fish keratocytes reveals two different mechanisms for generating a retrograde flow of actin. Mol. Biol. Cell 16, 507-518 (2005).

23. Lin, C. H. \& Forscher, P. Growth cone advance is inversely proportional to retrograde F-actin flow. Neuron 14, 763-771 (1995).

24. Mitchison, T. \& Kirschner, M. Cytoskeletal dynamics and nerve growth. Neuron 1 , 761-772 (1988)

25. Thery, M. et al. Anisotropy of cell adhesive microenvironment governs cell internal organization and orientation of polarity. Proc. Natl Acad. Sci. USA 103, 19771-19776 (2006). 


\section{METHODS}

Mouse strains. Mice with constitutive or conditional depletion of integrins (Itg) were intercrossed with $\mathrm{Mx} 1 \mathrm{Cre}$ transgenic mice, and have been described previously ${ }^{5}$. In this study, the following mice were used: $\operatorname{Tln}^{-/-}\left(\operatorname{talin} 1^{-1-} \mathrm{MxCre}\right), \operatorname{Itgb2} 2^{-1-}$,

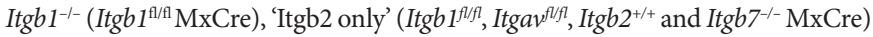
and $\operatorname{Itg}^{-/}\left(\operatorname{Itg} b 1^{f l / f l}, \operatorname{Itgav^{fl/fl}}, \operatorname{Itg} b 2^{+/+}\right.$and $\operatorname{Itg} b 7^{-/-}$MxCre). Integrin-deficient and wild-type mouse lines were all kept on a mixed 129SV/C57BL/6 genetic background. The mice were bred in a conventional animal facility at the Max Planck Institute of Biochemistry, according to the local regulations.

The induction of the hematopoietic knockout was performed as described recently ${ }^{5}$. Briefly, at an age between 8 and 12 weeks, mice of the desired genotype received a single intraperitoneal injection of $250 \mu \mathrm{g}$ Poly (I) Poly (C) (Amersham Biosciences), diluted in $0.5 \mathrm{ml}$ phosphate buffered saline (PBS). Mice were killed 10-14 days after injection and the knockout efficiency of hematopoietic precursors was estimated by flow cytometric analysis of peripheral blood platelets.

Cell generation, separation and purification. Bone marrow-derived dendritic cells and bone macrophages were generated from flushed bone marrow suspension as described previously ${ }^{26,27}$. For dendritic cells, at day 8-10 of culture, $200 \mathrm{ng} \mathrm{ml}^{-1}$ LPS (Sigma-Aldrich; E. coli 0127:B8) was added overnight and the Itgb $1^{-/-}$, 'Itgb2 only' and $\operatorname{Itg}^{-1-}$ dendritic cells were subsequently depleted of remaining $\mathrm{Gr}-1^{+}$and integrin $\beta 1^{+}$contaminants. For depletion, cells were incubated with biotinylated antibodies against Gr-1 (RB6-8C5) and integrin $\beta 1$ (Ha2/5; both BD Pharmingen), followed by streptavidin-microbead separation, according to the manufacturer's protocol (Miltenyi Biotech). The negatively selected dendritic cells used for migration assays were $>99 \%$ enriched for $\beta 1$ integrin-knockout cells, and $\beta 1$-deficient cells were always $100 \%$ deleted for integrin $\alpha v$. $\operatorname{Th}^{-1-}$ dendritic cells were generated identically and depleted of $\mathrm{Gr}-1^{+}$contaminants. To determine knockout efficiencies, dendritic cell lysates were loaded on $12 \%$ gradient gels for electrophoresis under reducing conditions and blotted. Talin was detected with a mouse anti-pan-talin antibody (T3287, Sigma), and the loading control was performed with rabbit anti-mouse actin antibody (A2066, Sigma).

Plasmid transfection and membrane labelling. For transfection with LifeactGFP, actin-GFP or MLC-GFP (a gift from M. Olson, Beatson Institute, Glasgow, UK), bone marrow-derived dendritic cells or macrophages (both between days 8-10 of culture) were transfected using the primary mouse T cell kit (Amaxa) and the Amaxa nucleoporator according to the manufacturer's recommendations. After transfection, $200 \mathrm{ng} \mathrm{ml}^{-1}$ LPS (Sigma-Aldrich; E. coli 0127:B8) was added to dendritic cells for overnight maturation. Before usage in chemotaxis assays, the cells were sorted for GFP-positive cells with a FACSAria (Beckton Dickinson). Murine embryonic fibroblasts were stably transfected with LifeactGFP as described previously?

To label the plasma membrane, $5 \times 10^{5}$ LPS-stimulated dendritic cells were incubated with $1.25 \mu \mathrm{l}$ of Vybrant DiO labelling solution (Molecular Probes, Invitrogen) in $500 \mu \mathrm{l} \mathrm{serum}$-free medium for $10 \mathrm{~min}$ at $37^{\circ} \mathrm{C}$, and washed according to manufacturer's instructions.

Chemotaxis assay. For the under-agarose (UA)-assay, $2 \times$ HBSS buffer (Sigma), RPMI (Invitrogen) with 20\% fetal calf serum (FCS; Invitrogen) for dendritic cells and 5\% FCS for macrophages, and 4\% agarose (Invitrogen) were mixed in a 1:2:1 ratio and subsequently casted into a custom-made migration chamber and equilibrated at $37^{\circ} \mathrm{C}\left(5 \% \mathrm{CO}_{2}\right)$ for $30 \mathrm{~min}$. A hole was stamped into the agarose layer and filled with $0.6 \mu \mathrm{g} \mathrm{ml}^{-1} \mathrm{CCL} 19$ (R\&D Systems). Within a distance of $5 \mathrm{~mm}$ 10,000 cells were injected between the glass coverslip and the agarose layer.

Polyethylene glycol (PEG) coating was performed as described previously ${ }^{28}$. Briefly, glass coverslips were immersed in a $1 \mathrm{mM}$ solution of a linear PEG,
$\mathrm{CH} 3-(\mathrm{O}-\mathrm{CH} 2-\mathrm{CH} 2) 43-\mathrm{NH}-\mathrm{CO}-\mathrm{NH}-\mathrm{CH} 2-\mathrm{CH} 2-\mathrm{CH} 2-\mathrm{Si}(\mathrm{OEt}) 3$, in dry toluene under a nitrogen atmosphere for $20 \mathrm{~h}$. Finally, the substrates were rinsed intensively with ethyl acetate, methanol and water, and dried with nitrogen. Serum/ PEG patterns were generated by coating glass coverslips with PEG and then scratching and incubating with serum containing Alexa 555-BSA (Invitrogen). For the poly-L-lysine coating, $10 \mu \mathrm{l}$ of $0.01 \%$ poly-L-lysine (Calbiochem) was added to the glass surface, which was incubated for $30 \mathrm{~min}$ at $37^{\circ} \mathrm{C}$, washed twice with PBS and dried for $30 \mathrm{~min}$ at room temperature. Concanavalin A (ConA) coating was achieved by incubating glass surfaces with $20 \mu \mathrm{l}$ of a $2 \mathrm{mg} \mathrm{ml}^{-1}$ stock solution of ConA (Calbiochem) for $5 \mathrm{~min}$ at room temperature, before brief air drying.

Live-cell imaging. Time-lapse bright-field movies of UA-assays were recorded at the indicated time intervals using inverted Axiovert 40 (Zeiss) cell culture microscopes and PAL cameras (Prosilica) triggered by custom-made software (SVS Visitek). Up to three samples were analysed simultaneously using three individual setups, thereby allowing the same conditions for controls and probes. Interference reflection microscopy (IRM) was carried out with a Leica TCSSP2 confocal microscope at 488-nm excitation wavelength. Total internal reflection (TIRF) and differential interference contrast (DIC) microscopy were performed with a Axiovert 200 (Zeiss) microscope, a TIRF 488/561-nm laser system (Visitron systems) and a Cool-Snap-HQ camera (Roper scientific) triggered by Metamorph software (Molecular Devices). Fluorescence recovery after photobleaching (FRAP) measurements were performed with an Axiovert 200 (Zeiss) microscope, a CSU10 spinning disc confocal scanhead (Yokogawa) and a Cool-Snap-HQ2 camera (Roper scientific), triggered by Metamorph software (Molecular Devices). All microscopes were equipped with custom-built climate chambers $\left(5 \% \mathrm{CO}_{2}, 37^{\circ} \mathrm{C}\right.$, humidified) and optionally with custom-built objective heating systems.

Cell directionality, cell speed, membrane flow and actin dynamics analysis. Manual cell tracking was carried out using ImageJ and the MTrackJ plugin (http:// www.imagescience.org/meijering/software/mtrackj/) or the Manual Tracking plugin (http://rsbweb.nih.gov/ij/). Cells were generally tracked for at least $1 \mathrm{~h}$ at $2 \mathrm{~min}$ per frame. Speed and directionality parameters were calculated and visualized by analysing the acquired data with the Chemotaxis And Migration Tool plugin (http://www.ibidi.de/applications/ap_chemo.html). Actin dynamics and membrane flow were analysed by kymograph and line-scan analysis, using Metamorph. The actin polymerization rate is the sum of the retrograde actin flow and the actin forward protrusion at the leading edge.

Image processing. Image processing was carried out with Metamorph Software. Kymographs were generated after processing of the image sequence with the flatten background function.

Statistical analysis. Student's $t$-tests and analysis of variance (ANOVA) were performed after data were confirmed to fulfill the criteria of normal distribution and equal variance, otherwise Kruskal-Wallis tests or Mann-Whitney U-tests were applied. If overall ANOVA or Kruskal-Wallis tests were significant, we performed a post hoc test. Analyses were performed with Sigma Stat 2.03. For all statistical details, see Supplementary Information, Tables S1 and S2.

26. Lutz, M. B. et al. An advanced culture method for generating large quantities of highly pure dendritic cells from mouse bone marrow. J. Immunol. Methods 223, 77-92 (1999).

27. Rutherford, M. S. \& Schook, L. B. Differential immunocompetence of macrophages derived using macrophage or granulocyte-macrophage colony-stimulating factor. J. Leukoc. Biol. 51, 69-76 (1992).

28. Blummel, J. et al. Protein repellent properties of covalently attached PEG coatings on nanostructured SiO(2)-based interfaces. Biomaterials 28, 4739-4747 (2007). 


\section{cell biology}

DOI: $10.1038 / \mathrm{ncb} 1992$
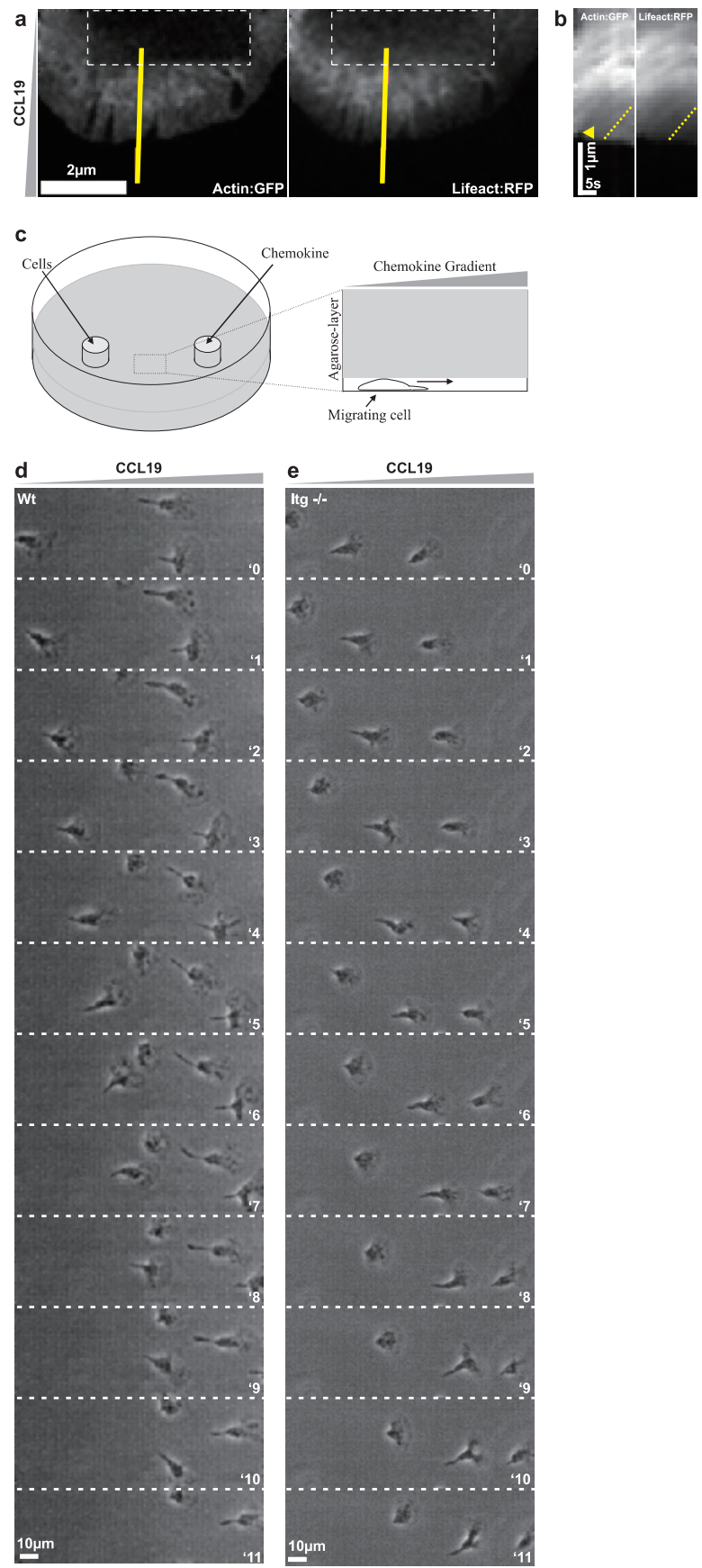

Figure S1 (a-b) Verification of actin flow patterns using photobleaching of actin:GFP and Lifeact:RFP in migrating dendritic cells (DCs). (a) Actin:GFP and Lifeact:RFP double-transfected DC migrating under agarose in a CCL19 gradient imaged with a spinning disc confocal microscope. The region in the dotted box was bleached with a 405nm focused laser beam, leading to bleaching of the actin:GFP signal whereas Lifeact:RFP recovers almost immediately. (b) Kymographic analysis of (a) (yellow line); the retrograde actin flow directly at the very leading edge is highlighted by a dashed yellow line and the bleached actin:GFP is highlighted by a yellow arrowhead, demonstrating the same pattern of the actin:GFP speckle signal, the bleached actin:GFP signal and the Lifeact:RFP signal. (c-d) Undisturbed migration of integrin deficient dendritic cells in under agarose assays. (c) Scheme of the experimental setup of the under agarose assay. (d) Wild-type (Wt) and integrin ${ }^{-/-}\left(\mathrm{Itg}^{--}\right)$dendritic cells migrating under agarose. ' $x$ indicates time in seconds. 

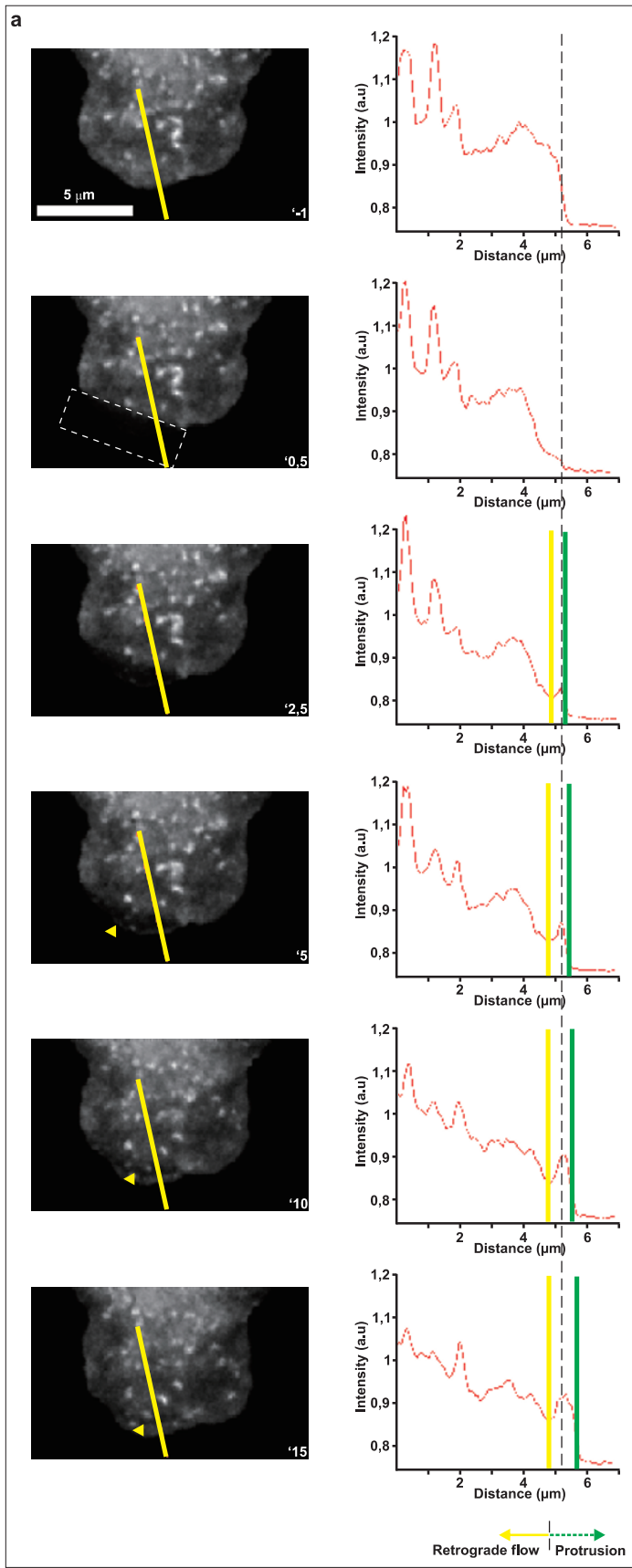

Figure S2 Verification of actin flow patterns using photobleaching of actin:GFP transfected dendritic cells (DCs). DCs migrated under agarose in CCL19 gradients and were imaged with a spinning disc confocal microscope. (a) Fluorescence recovery after photobleaching: the region in the dotted box was bleached with a $405 \mathrm{~nm}$ focused laser beam. Yellow arrowheads mark the bleached area that undergoes retrograde movement, while the leading edge advances over time. The right panels show intensity scans of the area marked with the yellow line in the left panels. The dotted grey line in the intensity diagram demarks the site of initial photobleaching, the green bars indicate the position of the leading edge, yellow bars the position of the bleached area. Green and yellow arrows on the bottom of the diagrams indicate the advancement of the leading edge (protrusion) and the retrograde slippage of the actin cortex (retrograde flow). Note that the bleached area moves backward almost without recovering in intensity, indicating that actin polymerization
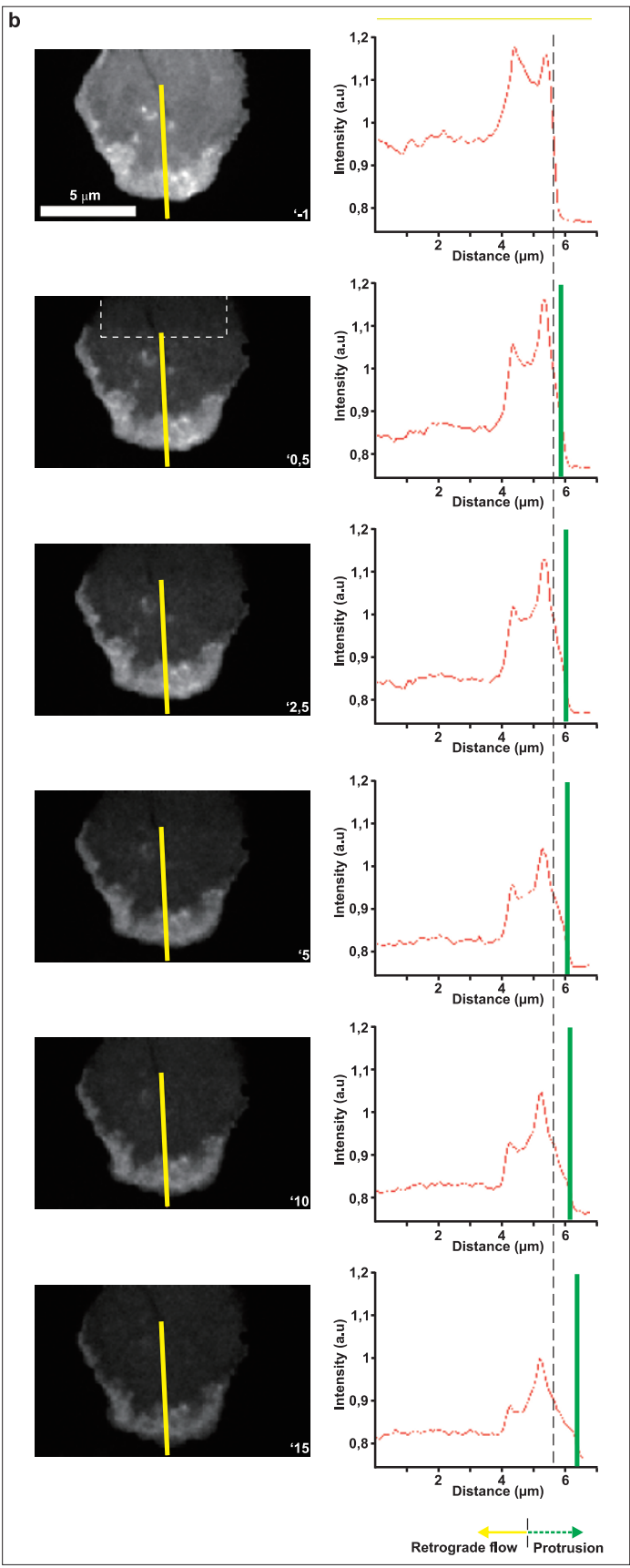

occurs predominantly at the leading edge. (b) A fluorescent localization after photobleaching type of experiment to exclude that bleaching directly at the leading edge induces an artificial behavior. The region in the dotted box was bleached as in (a). Yellow arrowheads demarcate the line where the first bleached G-actin monomers are incorporated into the leading edge cortex. Subsequently this line moves backward with the retrograde flow while the leading edge advances. Right panels show intensity scans of the area marked with a yellow line in the left panels. The grey dotted line in the intensity diagrams indicates the position of the leading edge at the time point of bleaching. The green line marks the position of the advancing leading edge, the yellow line the area where the first bleached monomers are incorporated. Green and yellow arrows on the bottom of the diagrams indicate the advancement of the leading edge (protrusion) and the retrograde slippage of the actin cortex (retrograde flow). ' $x$ indicates time in seconds. a.u.: arbitrary units. 

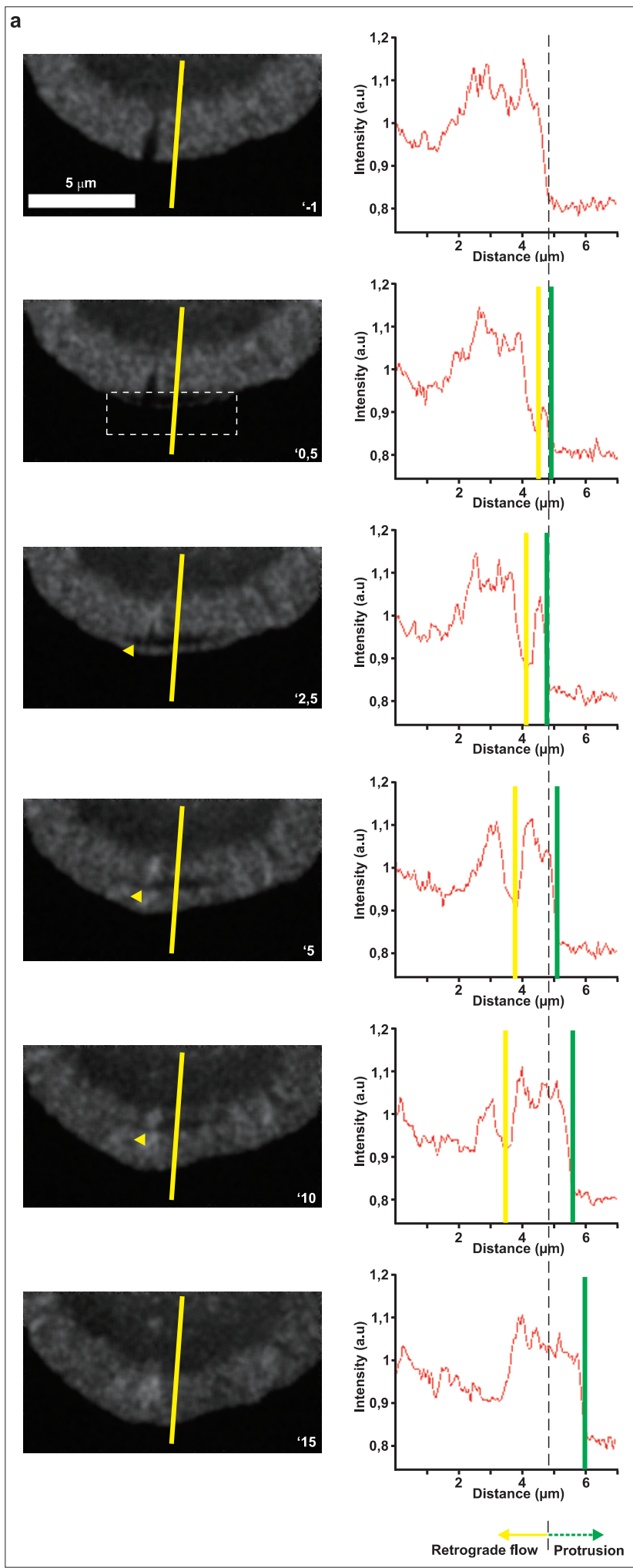

Figure S3 Verification of actin flow patterns on the inert substrate polyethylene glycol (PEG) using photobleaching of actin:GFP transfected dendritic cells (DCs). DCs migrated under agarose in CCL19 gradients on PEG coated substrates and were imaged with a spinning disc confocal microscope. (a) Fluorescence recovery after photobleaching: same
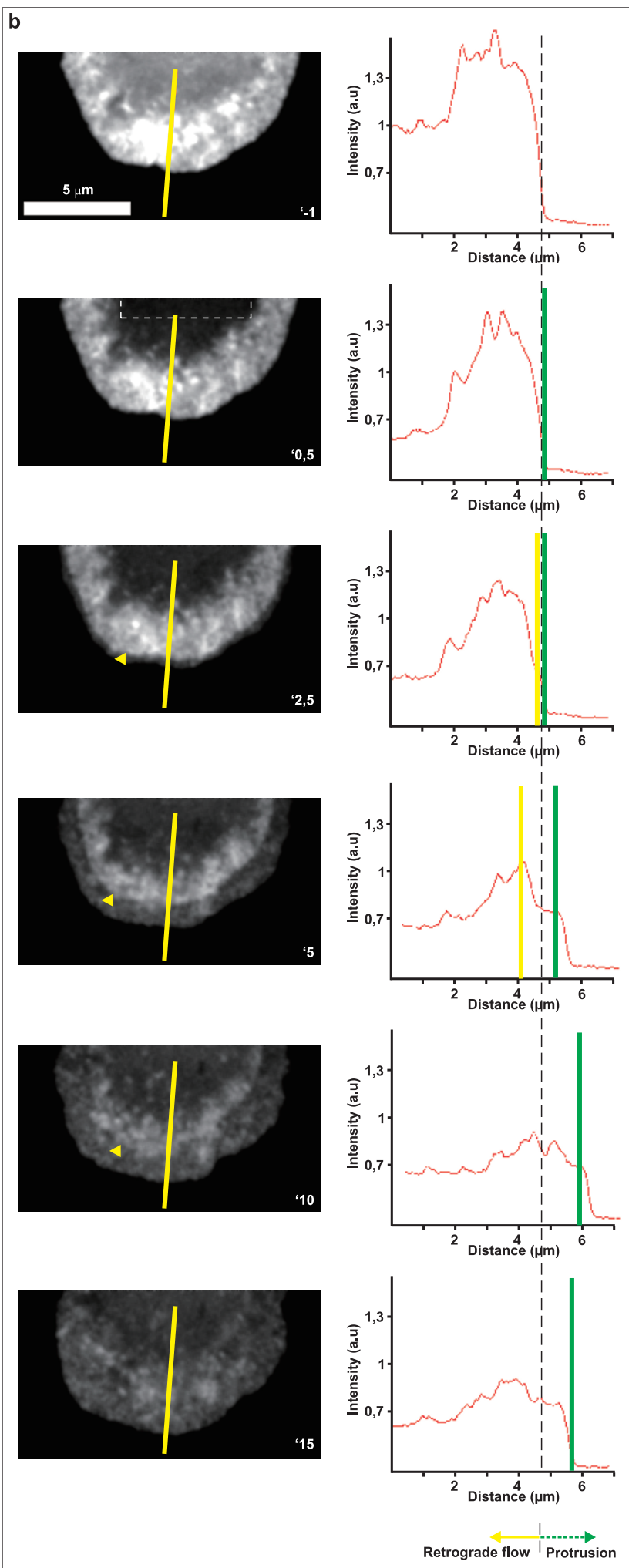

experimental set-up and figure labelling as in Fig. S2a. (b) A fluorescent localization after photobleaching type of experiment to exclude that bleaching directly at the leading edge induces artificial behavior: same experimental set-up and figure labelling as in Fig. S2b. ' $x$ indicates time in seconds. a.u.: arbitrary units. 

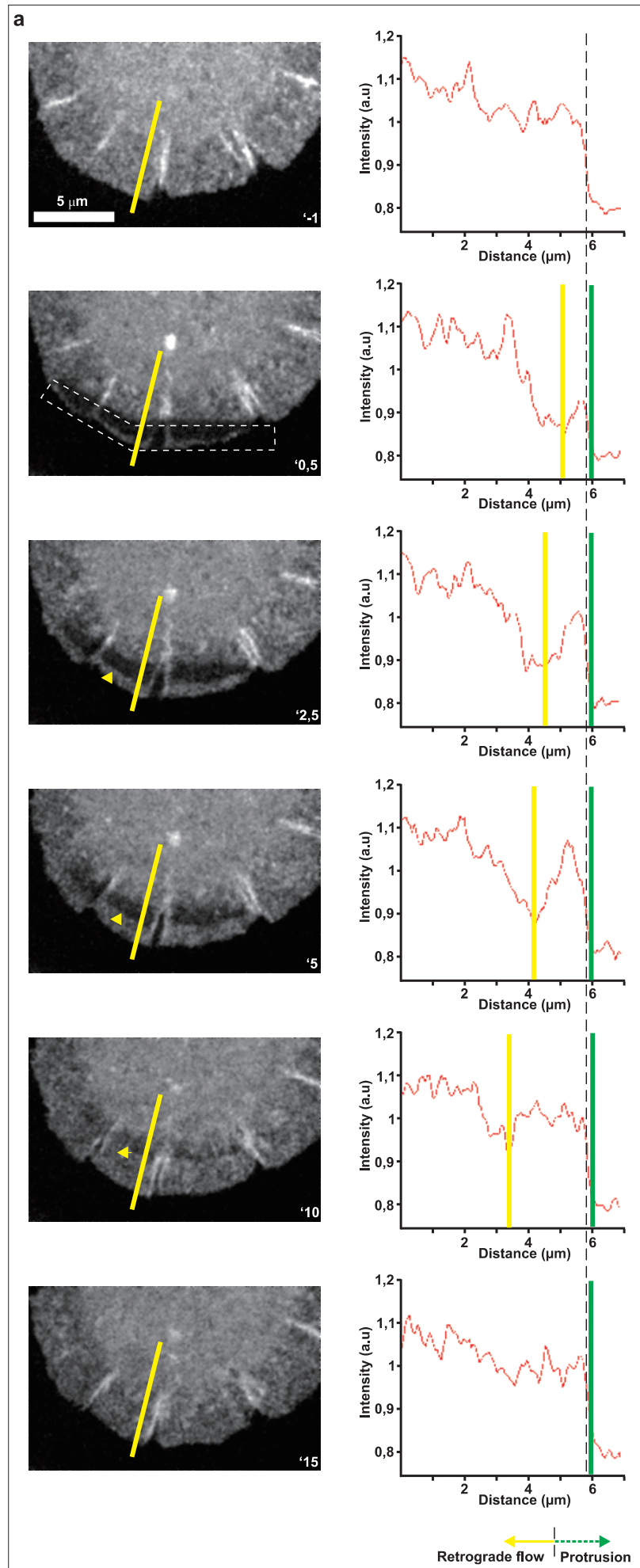

Figure S4 Verification of actin flow patterns in $\mathrm{Itg}^{-/}$dendritic cells using photobleaching of actin:GFP transfected Itg $^{-/-}$dendritic cells (DCs). DCs migrated under agarose in CCL19 gradients and were imaged with a spinning disc confocal microscope. (a) Fluorescence recovery after photobleaching: same experimental set-up and figure labelling
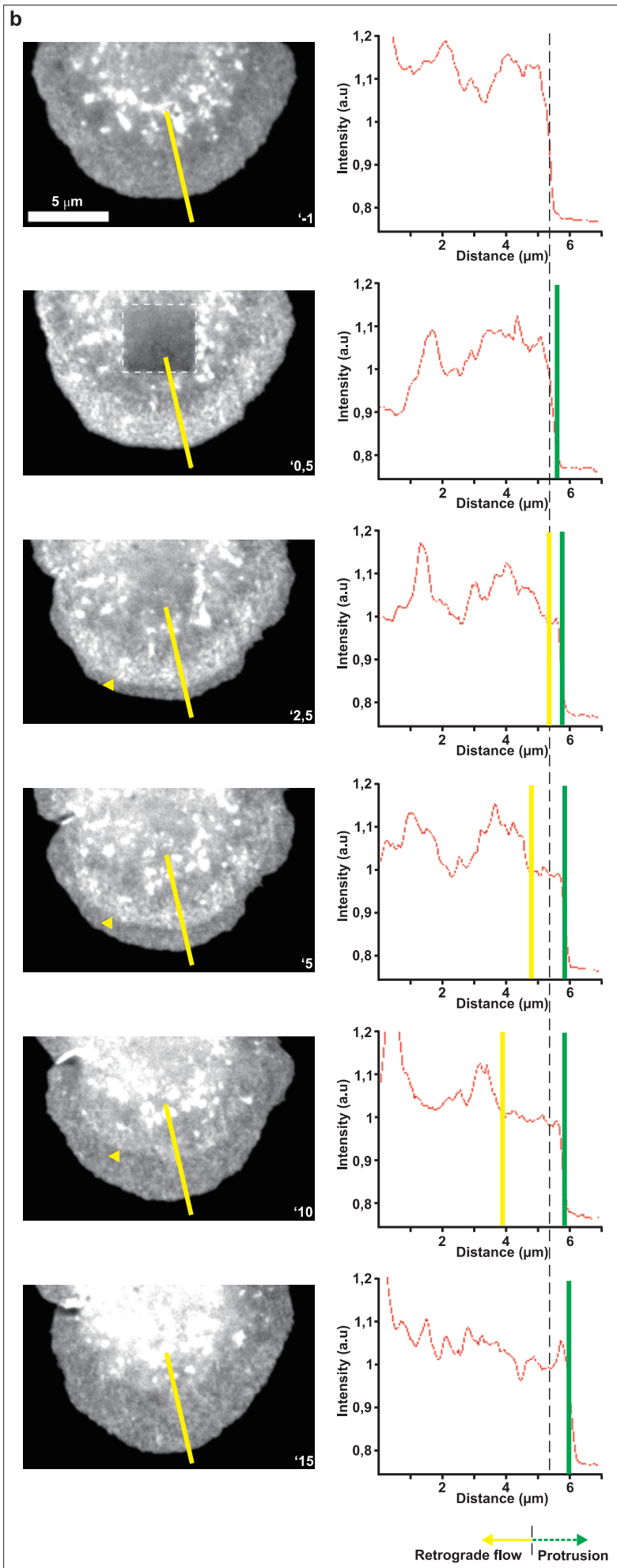

as in Fig. S2a. (b) A fluorescent localization after photobleaching type of experiment to exclude that bleaching directly at the leading edge induces an artificial behavior: same experimental set-up and figure labelling as in Fig. S2b. ' $x$ indicates time in seconds. a.u.: arbitrary units. 

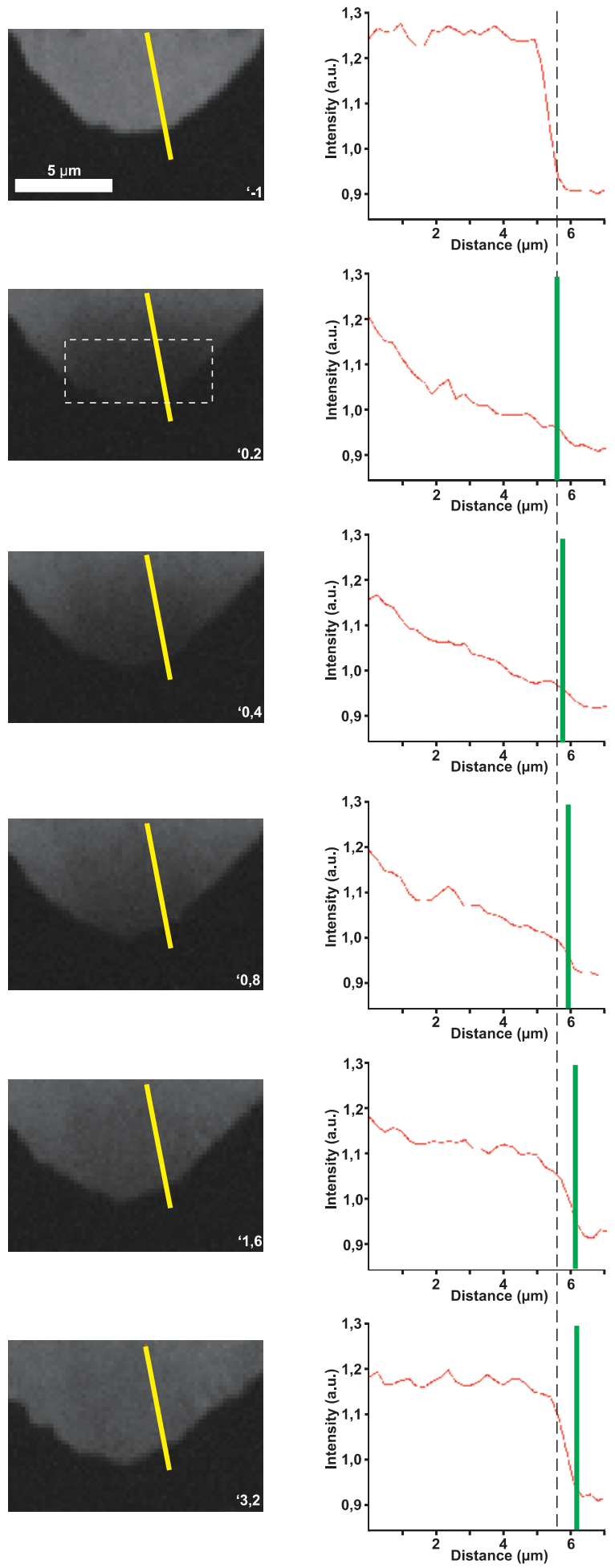

Figure S5 Membrane flow dynamics following photobleaching of the leading edge of dendritic cells (DCs) labelled with a membrane dye. DCs labelled with the plasma membrane dye DiO migrated under agarose in CCL19 gradients on a PEG coated glass substrate and were imaged with a spinning disc confocal microscope. Fluorescence recovery after photobleaching (FRAP): the region in the dotted box was bleached with a $405 \mathrm{~nm}$ focused

laser beam and subsequent images were recorded every 200 ms. Right panels are line scans illustrating the recovery of fluorescence intensity along the yellow line indicated in the left panels. The grey dotted line in the intensity diagrams indicates the position of the leading edge at the time point of bleaching. The green line marks the position of the advancing leding edge. ' $x$ indicates time in seconds. a.u.: arbitrary units. 

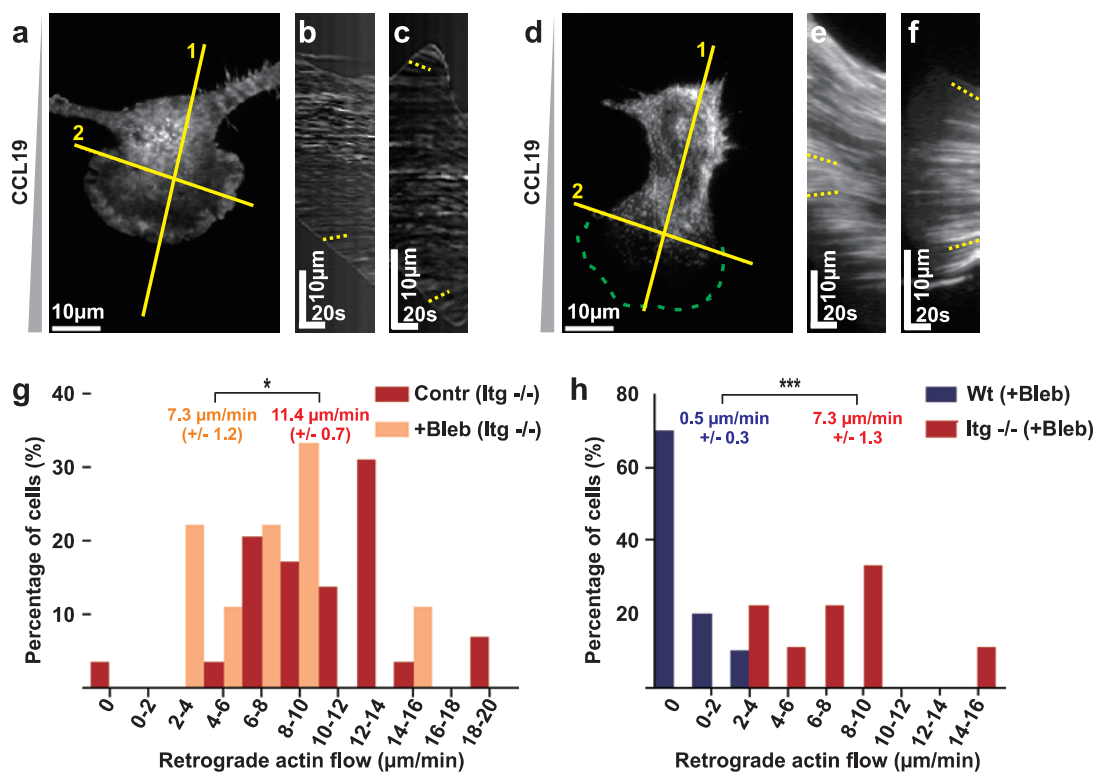

Figure S6 (a-f) The movement of MLC:GFP follows the same principal trajectories as F-actin and MLC-patches gradually converged at the trailing edge, reflecting their contractile activity. (a) Lifeact:GFP transfected dendritic cell (DC) migrating under agarose in a CCL19 gradient. (b) Kymographic analysis through the vertical cell axis (yellow line 1 in (a)).

(c) Kymographic analysis along the horizontal cell axis (yellow line 2 in (a)).

(d) MLC:GFP transfected DC migrating under agarose in a CCL19 gradient.

(e) Kymographic analysis along the vertical cell axis (yellow line 1 in (d)).

(f) Kymographic analysis along the horizontal cell axis (yellow line 2 in

(d)). Flow directions are highlighted by dashed yellow lines. MLC: myosinlight chain. (g-h) Retrograde flow in the absence of integrins is not caused by increased myosin II dependent contractility. Quantitative analysis of retrograde flow velocities of dendritic cells (DCs) migrating under agarose in CCL19 gradients. (g) Blebbistatin $(50 \mu \mathrm{M})$ treated vs. untreated integrin ${ }^{-/-}$ $\left(\operatorname{ltg}^{-/}\right)$DCs migrating under agarose $\left(n_{\text {Contr }}=30 ; n_{\text {Bleb }}=9\right)$. (h) Blebbistatin treated wild-type $(\mathrm{Wt})$ vs. Itg-- DCs migrating under agarose $\left(\mathrm{n}_{\mathrm{Wt}}=10 ; \mathrm{n}_{\text {Itg- }}\right.$ $=9$ ). Numbers indicate mean values +/- SD (in brackets). For statistical analysis see Supplementary Information, table S1. 

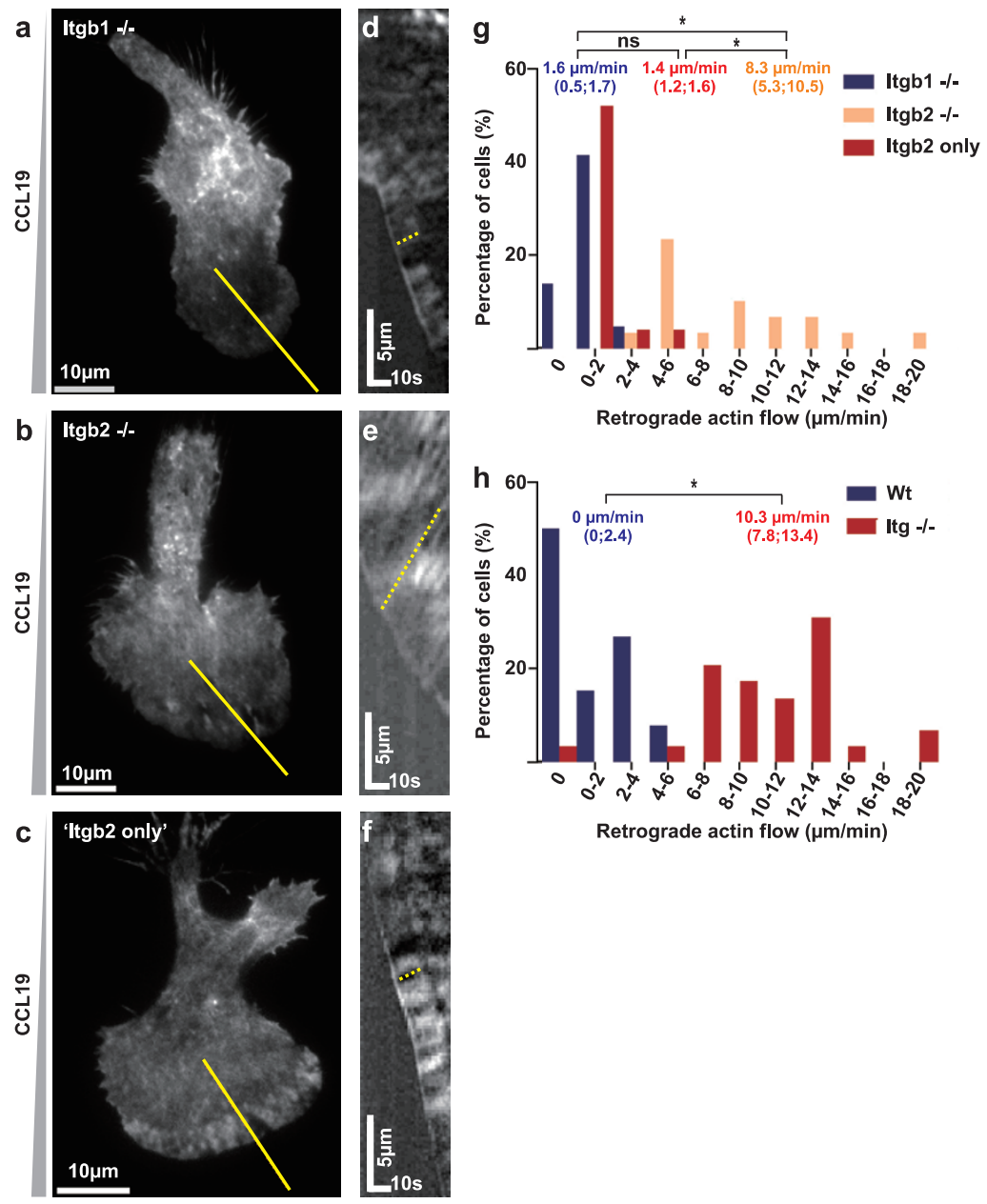

Figure S7 $\beta 2$ integrins mediate transmembrane force coupling in the under agarose assay. Quantitative analysis of retrograde flow velocities of dendritic cells migrating under agarose in CCL19 gradients. Cells of the following genotypes were analyzed: (a, d) $\operatorname{Itgb} 1^{-1}$; (b, e) $\operatorname{ltgb} 2^{-1-}$; (c, f) "Itgb2 only", meaning cells that express only $\beta 2$ integrins and no other integrins. (a-c) show images with the yellow line indicating the area displayed as a

kymograph in (D-F). Here the dotted lines mark retrograde flow velocities. (g) shows the quantitative analysis in comparison to the data sets of integrindeficient ( $\mathrm{Itg}^{-1-}$ ) and wild-type (Wt) cells (displayed in Fig. 2). ns: not significant; Single asterisks always denote $P<0.05$. Numbers in brackets in $\mathrm{G}$ and $\mathrm{H}$ represent median, $25 \%$ and $75 \%$ percentile $\left(\mathrm{n}_{\text {|tgb1-l/ }}=13\right.$; $\mathrm{n}_{\text {|tgb2-/- }}$ $=32 ; n_{\text {ltgb2only }}=15$ ). For statistical analysis see tables S1 and S2. 
a

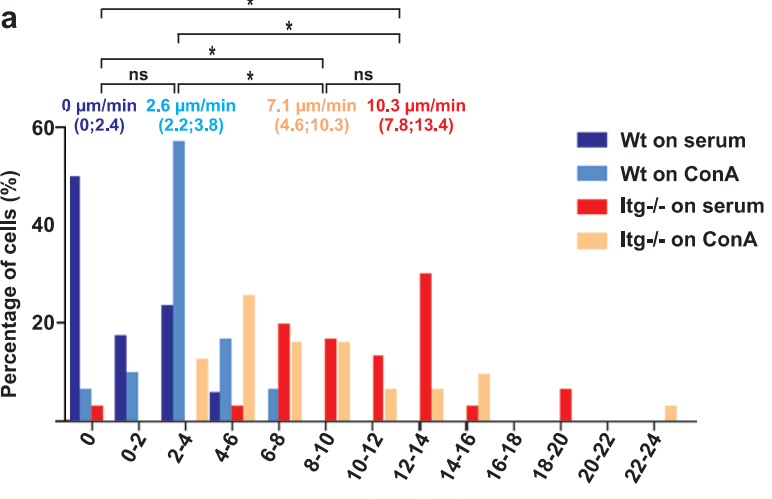

b

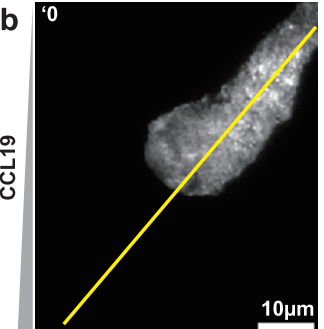

50
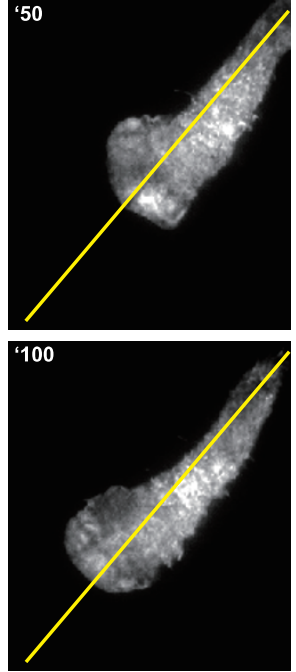

'150

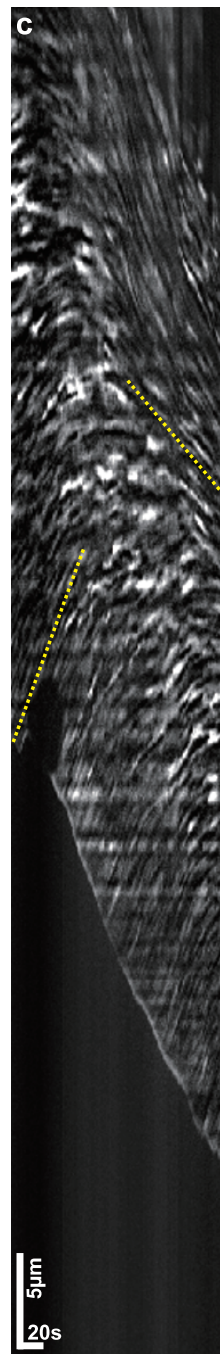

Figure $\mathbf{S 8}$ (a) Indication that the friction between actin cortex and membrane is mainly due to force coupling via integrins. Quantitative analysis of retrograde flow velocities of wild-type (wt) and $\mathrm{Itg}^{-/-}$dendritic cells (DCs) migrating under agarose in CCL19 gradients on serum or on ConcavalinA (ConA). DCs were unable to migrate ConA as the membrane was "glued" to the surface due to the unspecific binding of membrane carbohydrates to ConA (not shown). Note that actin retrograde flow rates are similar between wt DCs on serum coated surfaces and wt DCs on ConA, and similar between

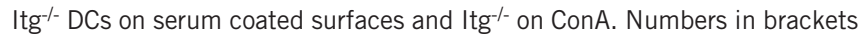
represent median, $25 \%$ and $75 \%$ percentile $\left(n_{W t S e r u m}=33 ; n_{W t C o n A}=29\right.$; $n_{\text {Itg-/-Serum }}=29 ; n_{\text {Itg-l-ConA }}=30$ ). For statistical analysis see Supplementary Information, table S1. (b-c) Kymograph analysis of a dendritic cell migrating on polyethylene glycol (PEG), highlighting the convergence zone of actin flow. (b) Time-lapse sequence, the yellow line marks the region depicted in (c) as a kymograph. (c) Kymographic analysis of (b), the actin flow directions are highlighted by yellow dotted lines. ' $x$ indicates time in seconds. 

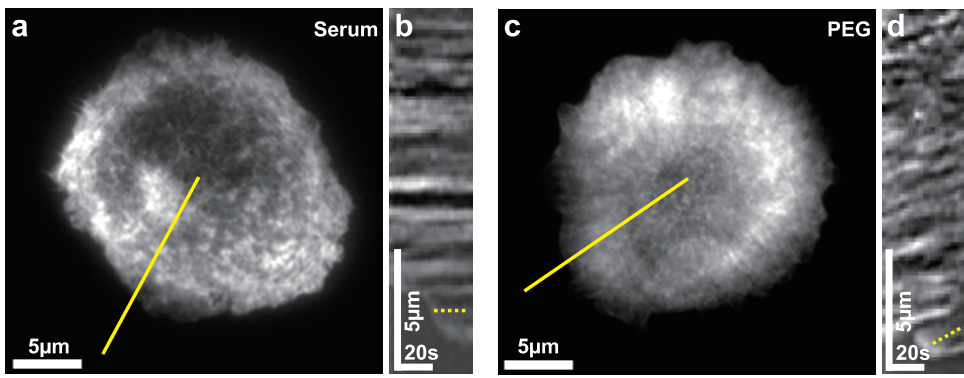

e
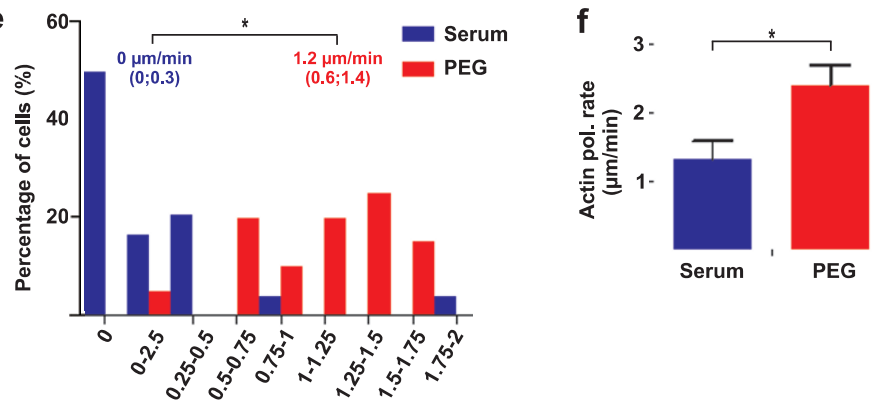

Retrograde actin flow $(\mu \mathrm{m} / \mathrm{min})$

g

F-actin bundle

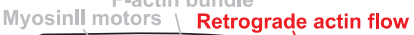

Coupled

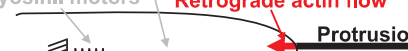

Mlecular

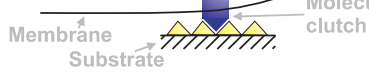

Substrate

Myosinll motors Retrograde actin flow

Slipping

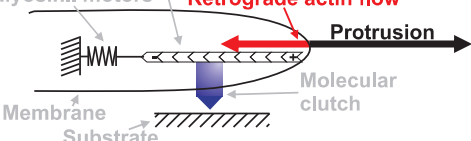

Substrate

F-actin bundlle

Myosinll motors Retrograde actin flow

Uncoupled

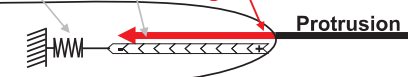

Substrane

77

7777.

Figure S9 (a-d) Increased actin polymerization rate in macrophages on polyethylene glycol (PEG) vs. serum coated surfaces. Lifeact:GFP transfected macrophage in the under-agarose-assay (a) on a serum-coated surface or (c) on PEG. (b) and (d) show the kymographic analysis of the yellow line regions in (a) and (c), respectively, the actin retrograde flow is highlighted by yellow dotted lines. (e) Quantitative analysis of retrograde flow velocities. Numbers in brackets represent median, $25 \%$ and $75 \%$ percentile $\left(n_{\text {Glas }}=23\right.$;
$\left.n_{P E G}=19\right)$. (f) Quantitative analysis of actin polymerization rates $\left(n_{G l a s}=9\right.$ $n_{P E G}=8$ ). For statistical analysis see Supplementary Information, table S1. (g) Schematic of protrusion and retrograde actin flow velocities upon different degrees of force coupling. Note that retrograde actin flow velocities enhance upon uncoupling whereas the protrusion velocity stays constant, leading to an enhanced actin polymerization rate (as the sum of protrusion and retrograde flow) at the leading edge. 


\section{Supplementary movie legends}

Movie S1 Comparison of retrograde actin flow patterns in Lifeact:RFP and actin:GFP double-transfected dendritic cells. Dendritic cells migrate under agarose towards a CCL19 gradient. Photobleaching in the centre leads to integration of bleached actin:GFP monomers directly at the cellular front with a subsequent backward movement (left panel), whereas Lifeact:RFP directly recovers (right panel). Spinning disc confocal microscopy, time-lapse over $32 \mathrm{~s}$ after photobleaching ( $2 \mathrm{~s} / \mathrm{frame})$, the scale bar is equal to $5 \mu \mathrm{m}$.

Movie S2 The pushing force of actin polymerization is not the sole force driving actin retrograde flow. The G-actin sequestering drug latrunculin is added to a Lifeact:GFP transfected dendritic cell adherent to poly-L-lysine. The membrane stays attached to the substrate, whereas the actin cytoskeleton moves into the cell upon latrunculin treatment, indicating that actin polymerization is not the sole force driving actin retrograde flow. The sequence shows the timepoints directly following latrunculin addition. TIRF microscopy, time-lapse over $200 \mathrm{~s}$ ( $1 \mathrm{~s} / \mathrm{frame}$ ), the scale bar is equal to $5 \mu \mathrm{m}$.

Movie S3 Actin polymerization and myosin II activity are the main forces acting onto the actin cytoskeleton of dendritic cells. Before the addition of the actin-polymerization-inhibiting drug latrunculin to a Lifeact:GFP transfected dendritic cell adherent to poly-L-lysine, the myosin- inhibiting drug Blebbistatin is added. This pretreatment leads to an almost complete stalling of actin dynamics, indicating that actin polymerization and myosin II activity are the main forces acting onto the actin cytoskeleton of dendritic cells. The sequence shows the timepoints directly before, 10 min after Blebbistatin addition and directly following latrunculin addition (time-lapse over 38s, 30s and 34s, respectively). TIRF microscopy, $1 \mathrm{~s} /$ frame, the scale bar is equal to $10 \mu \mathrm{m}$.

Movie S4 Spatial and temporal dynamics of the actin cytoskeleton in dendritic cells. Lifeact:GFP transfected dendritic cell migrates under agarose towards a CCL19 gradient. The cell shows a broad leading edge with a diffusely protruding actin-cortex and a trailing edge with thicker actin bundles. TIRF microscopy, time-lapse over $50 \mathrm{~s}$ ( $1 \mathrm{~s} / \mathrm{frame}$ ), the scale bar is equal to $10 \mu \mathrm{m}$.

Movie S5 Retrograde actin flow measurements in actin:GFP expressing dendritic cells by fluorescence recovery after photobleaching. Actin:GFP transfected dendritic cells migrate under agarose towards a CCL19 gradient. Photobleaching at the leading edge (left panel) leads to integration of actin monomers directly at the cellular front, whereas the bleached area moves backwards into the cell center. Photobleaching in the centre (right panel) leads to integration of bleached actin:GFP monomers directly at the cellular front with a subsequent backward movement. The sequence shows timepoints before and after photobleaching. Spinning disc confocal microscopy, time-lapse over $3,5 \mathrm{~s}$ before and $25 \mathrm{~s}$ after photobleaching $(0.5 \mathrm{~s} / \mathrm{frame})$, the scale bar is equal to $5 \mu \mathrm{m}$.

Movie S6 Membrane flow measurements in dendritic cells by fluorescence recovery after photobleaching. Dendritic cells are labelled with the fluorescent lipid dye DiO to label membrane lipids and migrate under agarose towards a CCL19 gradient on a PEG coated substrate. Photobleaching at the leading edge does not lead to a preferential recovery at the cell front. The sequence shows timepoints in pseudocolours (reflecting the distribution of fluorescence intensity) before and after photobleaching. Spinning disc confocal microscopy, time-lapse over 3,5s before and $25 \mathrm{~s}$ after photobleaching ( $0.5 \mathrm{~s} /$ frame), the scale bar is equal to $5 \mu \mathrm{m}$.

Movie S7 Spatial and temporal dynamics of myosin II in dendritic cells. A MLC-GFP transfected dendritic cell migrates under agarose towards a CCL19 gradient. The dot-like appearance of MLC with a density gradient decreasing from the cell rear to the front can be seen. The leading edge is completely devoid of signal and the MLC patches started to materialize 5-10 $\mu \mathrm{m}$ behind the leading front. TIRF microscopy, time-lapse over $120 \mathrm{~s}(2 \mathrm{~s} / \mathrm{frame})$, the scale bar is equal to $10 \mu \mathrm{m}$.

Movie S8 Spatial and temporal dynamics of the actin cytoskeleton in integrin-deficient dendritic cells. Lifeact:GFP transfected integrin ${ }^{-1-}$ dendritic cell migrates under agarose towards a CCL19 gradient. The actin retrograde is dramatically increased in comparison to wild-type dendritic cells, whereas protrusion velocity is unaffected. TIRF microscopy, time-lapse over $70 \mathrm{~s}$ ( $1 \mathrm{~s} /$ frame), the scale bar is equal to $10 \mu \mathrm{m}$.

Movie S9 Spatial and temporal dynamics of myosin II in integrin-deficient dendritic cells. MLC-GFP transfected integrin ${ }^{-/}$dendritic cell migrates under agarose towards a CCL19 gradient, displaying an enhanced retrograde movement of MLC in comparison to the MLC movement in wild-type dendritic cells. TIRF microscopy, time-lapse over 150 s ( $1 \mathrm{~s} /$ frame), the scale bar is equal to $10 \mu \mathrm{m}$.

Movie S10 Increased actin polymerisation rate and retrograde flow velocity upon uncoupling of the molecular clutch on an inert substrate. Lifeact:GFP transfected dendritic cell migrates under agarose towards a CCL19 gradient on an inert substrate (polyethylene glycol), displaying an enhanced retrograde actin flow. TIRF microscopy, time-lapse over $150 \mathrm{~s}$ ( $1 \mathrm{~s} /$ frame), the scale bar is equal to $10 \mu \mathrm{m}$.

Movie S11 Fibroblasts strictly adapt their shape to the adhesive surface, but form short protrusions onto the inert substrate which goes in hand with a massive retrograde actin flow. Lifeact:GFP transfected fibroblasts in the under agarose assay on patterned surfaces containing serum-coated tracks alternating with PEG-coated areas. The left panel shows the the Lifeact:GFP signal (and the border of the serum-coated track as a red line), the right panel shows the Alexa555-BSA signal marking the serum-coated tracks. TIRF microscopy, time-lapse over 100s ( $1 \mathrm{~s} /$ frame), the scale bar is equal to $5 \mu \mathrm{m}$. 


\section{Table S1.}

\section{Statistical analyses}

T-tests and ANOVAs were performed after data were confirmed to fulfill the criteria, otherwise Kruskal-Wallis tests or Mann-Whitney U-tests were applied. If overall ANOVA or Kruskal-Wallis tests were significant, we performed posthoc tests with pair-wise comparison (ANOVA: Tukey, Kruskal-Wallis: Dunn).

Analyses were performed with Sigma Stat 2.03.

\begin{tabular}{|c|c|c|c|c|}
\hline Fig. & Test statistic & $\begin{array}{l}\text { Sample size I } \\
\text { degrees of freedom }\end{array}$ & P-value & Posthoc test \\
\hline $1 \mathrm{~b}$ & t-test; $t=0.53$ & $d f=9$ & $P=0.61$ & \\
\hline $1 d$ & $\begin{array}{l}\text { ANOVA; } \\
F=92.1\end{array}$ & $\mathrm{df}=2.29$ & $P<0.001$ & $\begin{array}{l}\text { Tukey, all } \\
\text { pairwise } \\
P<0.001\end{array}$ \\
\hline $2 \mathrm{j}$ & U-test; $T=21$ & $\mathrm{n} 1=6, \mathrm{n} 2=16$ & $P<0.001$ & \\
\hline $2 \mathrm{k}$ & t-test; $\mathrm{t}=0.774$ & $d f=60$ & $P=0.442$ & \\
\hline 21 & t-test; $t=8.7$ & $d f=60$ & $P<0.001$ & \\
\hline $\begin{array}{l}2 \mathrm{~g}, 3 \mathrm{e}, \\
\text { S7g, } \\
\text { S7h }\end{array}$ & $\begin{array}{l}\text { Kruskal- } \\
\text { Wallis; } \\
H=117.18\end{array}$ & $d f=6$ & $P<0.001$ & $\begin{array}{l}\text { Dunns, all } \\
\text { pairwise, for } \\
\text { details see } \\
\text { table S2 }\end{array}$ \\
\hline $3 f$ & t-test; $t=3.2$ & $d f=54$ & $P=0.002$ & \\
\hline $3 g$ & $\begin{array}{l}\text { Spearman } \\
\text { Rank } \\
\text { correlation; } \\
r=0.12\end{array}$ & $n=33$ & $P=0.52$ & \\
\hline $4 d$ & $\mathrm{t}$-test; $t=5$, & $\mathrm{df}=12$ & $P<0.001$ & \\
\hline S6Ba & t-test; $t=2.786$ & $d f=37$ & $P<0.05$ & \\
\hline $\mathrm{S} 6 \mathrm{Bb}$ & t-test; $t=5.496$ & $d f=17$ & $P<0.001$ & \\
\hline S8A & $\begin{array}{l}\text { Kruskal- } \\
\text { Wallis; } \\
H=82.372\end{array}$ & $d f=3$ & $P<0.001$ & $\begin{array}{l}\text { Dunns, all } \\
\text { pairwise, } \\
P<0.05\end{array}$ \\
\hline S9Ae & $\begin{array}{l}\text { Mann- } \\
\text { Whitney; } \\
\mathrm{T}=594.000\end{array}$ & $\begin{array}{l}\mathrm{n}(\mathrm{small})=19 \mathrm{n}(\mathrm{big})= \\
23\end{array}$ & $P<0.001$ & \\
\hline S9Af & $\begin{array}{l}\text { t-test; } t=- \\
2.732\end{array}$ & $d f=15$ & $P<0.05$ & \\
\hline
\end{tabular}




\section{Table S2.}

Posthoc tests of Figs. $2 \mathrm{~g}, 3 \mathrm{e}$, suppl. fig. $7 \mathrm{~g}, 7 \mathrm{~h}$

Kruskall Wallis; posthoc all pairwise comparison (Dunn's Method):

\begin{tabular}{|c|c|}
\hline COMPARISON & P-value \\
\hline $\mathrm{Itg}^{-1-}$ vs Wt & $P<0.05$ \\
\hline $\operatorname{Itg}^{-1-}$ vs $\operatorname{TIn}^{-1-}$ & $P<0.05$ \\
\hline Itg $^{-1 /}$ vs Itgb2 only & $P<0.05$ \\
\hline $\mathrm{Itg}^{-1-}$ vs Wt on PEG & $P<0.05$ \\
\hline $\operatorname{Itg}^{-1-}$ vs $\mathrm{TIn}^{-1-}$ & $\mathrm{ns}$ \\
\hline $\operatorname{ltg}^{--/}$vs Itgb2 $2^{-1-}$ & $\mathrm{ns}$ \\
\hline Itgb2 ${ }^{-1-}$ vs Wt & $P<0.05$ \\
\hline $\operatorname{ltg} b 2^{-1-}$ vs Itgb $1^{-1-}$ & $P<0.05$ \\
\hline Itgb2 ${ }^{-1-}$ vs Itgb2 only & $P<0.05$ \\
\hline $\operatorname{Itgb2}^{-1-}$ vs Wt on PEG & $P<0.05$ \\
\hline $\operatorname{ltgb2} 2^{-1-}$ vs $\operatorname{TIn}^{-1-}$ & $\mathrm{ns}$ \\
\hline $\mathrm{Tln}^{-1-}$ vs Wt & $P<0.05$ \\
\hline $\operatorname{Tln}^{-1-}$ vs Itgb $1^{-1-}$ & $P<0.05$ \\
\hline TIn $^{-1-}$ vs Itgb2 only & $P<0.05$ \\
\hline TIn $^{-1-}$ vs WT on PEG & $\mathrm{ns}$ \\
\hline Wt on PEG vs WT & $P<0.05$ \\
\hline Wt on PEG vs Tlnn & $P<0.05$ \\
\hline Wt on PEG vs Itgb2 only & ns \\
\hline Itgb2 only vs Wt & $\mathrm{ns}$ \\
\hline Itgb2 only vs Itgb1 $1^{-1-}$ & ns \\
\hline $\operatorname{ltg} 11^{-1-}$ vs Wt & ns \\
\hline
\end{tabular}

